\title{
OPTIMALITY CONDITIONS AND DUALITY MODELS FOR MINMAX FRACTIONAL OPTIMAL CONTROL PROBLEMS CONTAINING ARBITRARY NORMS
}

\author{
G. J. ZALMAI
}

\begin{abstract}
Both parametric and parameter-free necessary and sufficient optimality conditions are established for a class of nondifferentiable nonconvex optimal control problems with generalized fractional objective functions, linear dynamics, and nonlinear inequality constraints on both the state and control variables. Based on these optimality results, ten Wolfe-type parametric and parameterfree duality models are formulated and weak, strong, and strict converse duality theorems are proved. These duality results contain, as special cases, similar results for minmax fractional optimal control problems involving square roots of positive semidefinite quadratic forms, and for optimal control problems with fractional, discrete max, and conventional objective functions, which are particular cases of the main problem considered in this paper. The duality models presented here contain various extensions of a number of existing duality formulations for convex control problems, and subsume continuous-time generalizations of a great variety of similar dual problems investigated previously in the area of finitedimensional nonlinear programming.
\end{abstract}

\section{Introduction}

In this paper, we shall establish necessary and sufficient optimality conditions and construct several parametric and nonparametric duality models for the following minmax fractional optimal control problem :

$$
\text { (P) Minimize } \max _{1 \leq i \leq T} \frac{\int_{a}^{b}\left[f_{i}(x(t), u(t), t)+\left\|K_{i}(t) x(t)\right\|_{k(i)}+\left\|L_{i}(t) u(t)\right\|_{\ell(i)}\right] d t}{\int_{a}^{b}\left[g_{i}(x(t), u(t), t)-\left\|M_{i}(t) x(t)\right\|_{m(i)}-\left\|N_{i}(t) u(t)\right\|_{n(i)}\right] d t}
$$

Received April 14, 2003.

2000 Mathematics Subject Classification: Primary 49K35, 49N15; Secondary $90 \mathrm{C} 32,90 \mathrm{C} 47$.

Key words and phrases: minmax fractional optimal control problems, constraints, arbitrary norms, optimality conditions, duality models, duality theorems. 
subject to

$$
\begin{gathered}
x(a)=0, \quad x(b)=0 \\
D x(t)=A(t) x(t)+B(t) u(t), \quad t \in[a, b],
\end{gathered}
$$

$$
h_{j}(x(t), u(t), t)+\left\|P_{j}(t) x(t)\right\|_{p(j)}+\left\|Q_{j}(t) u(t)\right\|_{q(j)} \leq 0, t \in[a, b], j \in \underline{s},
$$

$$
x \in C^{n}[a, b], \quad u \in P W S^{m}[a, b],
$$

where $C^{n}[a, b]$ is the space of all continuous $n$-dimensional vector functions $x:[a, b] \rightarrow \mathbb{R}^{n}$ (n-dimensional Euclidean space) defined on the compact interval $[a, b]$ of the real line $\mathbb{R}$, with the graph norm $\|x\|=$ $\|x\|_{\infty}+\|D x\|_{\infty}$, where the differentiation operator $D$ is given by

$$
y=D x \leftrightarrow x(t)=\int_{a}^{t} y(\tau) d \tau
$$

thus $D=d / d t$ except at the discontinuities of the piecewise smooth function $y:[a, b] \rightarrow \mathbb{R}^{n} ; P W S^{m}[a, b]$ is the space of all piecewise smooth $m$-dimensional vector functions defined on $[a, b]$, with the uniform norm $\|\cdot\| ; f_{i}, g_{i}, i \in \underline{r} \equiv\{1,2, \ldots, r\}$, and $h_{j}, j \in \underline{s}$, are continuously differentiable real-valued functions defined on $\mathbb{R}^{n} \times \mathbb{R}^{m} \times[a, b] ; A(t), B(t), K_{i}(t)$, $L_{i}(t), M_{i}(t), N_{i}(t), P_{j}(t)$, and $Q_{j}(t), i \in \underline{r}, j \in \underline{s}$, are, respectively, $n \times n, n \times m, k_{i} \times n, \ell_{i} \times m, m_{i} \times n, n_{i} \times m, p_{j} \times n$, and $q_{j} \times m$ matrices whose entries are continuous real-valued functions defined on $[a, b] ;\|\cdot\|_{k(i)},\|\cdot\|_{\ell(i)},\|\cdot\|_{m(i)},\|\cdot\|_{n(i)},\|\cdot\|_{p(j)}$, and $\|\cdot\|_{q(j)}, i \in \underline{r}, j \in \underline{s}$, are arbitrary norms, and for each $i \in \underline{r}$, the numerator of the inner objective function is nonnegative and its denominator is positive for all state-control pairs $(x, u)$ satisfying the constraints of $(\mathrm{P})$.

Finite-dimensional counterparts of $(\mathrm{P})$ are known as generalized fractional programming problems in the literature of mathematical programming. These problems have arisen in the areas of multiobjective programming [1], approximation theory $[2,3,14,24]$, goal programming $[7$, 13], and economics [23], among others.

The notion of duality for generalized linear fractional programming was initially considered by von Neumann [23] in his investigation of economic equilibrium problems. More recently, various optimality criteria, duality formulations, and computational algorithms for several classes of generalized linear and nonlinear fractional programming problems have appeared in the related literature. A fairly extensive list of references pertaining to various aspects of these problems is given in [30]. 
In contrast to the finite-dimensional case, infinite-dimensional problems of this type and, in particular, optimal control problems with generalized fractional objective functions have not yet received much attention in the literature of optimization theory and, consequently, at present no significant results of any kind are available for these problems. In fact, it appears that so far [25] and [29] are the only two papers which discuss certain aspects of this problem. Specifically, a Lagrangian-type dual problem was identified for $(\mathrm{P})$ in [25] with the help of a Gordantype theorem of the alternative, whereas a different dual problem along with saddle-point-type optimality criteria were presented in [29]. These results were obtained without assuming any differentiability conditions.

If in $(\mathrm{P})$ we set $r=1$, then the resulting special case is called a fractional optimal control problem. Some interesting applications of optimality conditions for problems of this type (without the norms) are discussed in [19-22].

In the present study, we shall establish, under suitable differentiability and convexity assumptions, both parametric and nonparametric necessary and sufficient optimality conditions, construct several parametric and parameter-free duality models, and prove appropriate duality theorems. Our approach to achieving these goals is based on a set of optimality conditions for a related problem discussed in [6], and two ancillary problems that are intimately linked to $(\mathrm{P})$.

The rest of this paper is organized as follows. In Section 2 we recall a set of necessary optimality conditions given in [6] for a special case of $(\mathrm{P})$. In Section 3 we utilize these optimality conditions in conjunction with some other auxiliary results to establish both parametric and nonparametric necessary optimality principles for $(\mathrm{P})$. We begin our discussion of duality for (P) in Section 4 where we introduce two parametric duality models and prove weak, strong, and strict converse duality theorems under appropriate convexity assumptions. In Sections 5 and 6 we formulate a total of eight parameter-free duality models for (P) and prove appropriate duality theorems. Finally, in Section 7 we briefly discuss an important special case of $(\mathrm{P})$ which involves square roots of positive semidefinite quadratic forms.

It is evident that all the results obtained for $(\mathrm{P})$ are also applicable, when appropriately specialized, to the following classes of optimal control problems with fractional, discrete max, and conventional objective functions, which are particular cases of $(\mathrm{P})$ :

$$
(P 1) \underset{(x, u) \in \mathbb{F}}{\operatorname{Minimize}} \frac{\int_{a}^{b}\left[f_{1}(x(t), u(t), t)+\left\|K_{1}(t) x(t)\right\|_{k(1)}+\left\|L_{1}(t) u(t)\right\|_{\ell(1)}\right] d t}{\int_{a}^{b}\left[g_{1}(x(t), u(t), t)-\left\|M_{1}(t) x(t)\right\|_{m(1)}-\left\|N_{1}(t) u(t)\right\|_{n(1)}\right] d t},
$$


$(P 2) \underset{(x, u) \in \mathbb{F}}{\operatorname{Minimize}} \max _{1 \leq i \leq r} \int_{a}^{b}\left[f_{i}(x(t), u(t), t)+\left\|K_{i}(t) x(t)\right\|_{k(i)}+\left\|L_{i}(t) u(t)\right\|_{\ell(i)}\right] d t$,

$$
\underset{(x, u) \in \mathbb{F}}{\operatorname{Minimize}} \int_{a}^{b}\left[f_{1}(x(t), u(t), t)+\left\|K_{1}(t) x(t)\right\|_{k(1)}+\left\|L_{1}(t) u(t)\right\|_{\ell(i)}\right] d t,
$$

where $\mathbb{F}$ (assumed to be nonempty) is the feasible set of $(\mathrm{P})$, that is,

$$
\mathbb{F}=\left\{(x, u) \in C^{n}[a, b] \times P W S^{m}[a, b]:(1)-(3) \text { hold }\right\} .
$$

Since in most cases the results established for $(P)$ can be modified and restated for (P1)-(P3) in a straightforward manner, we shall not explicitly state these results.

Although different concepts of duality have been discussed for various types of conventional variational and optimal control problems (see, e.g., $[11,15,16,17]$ and the references therein) constrained optimal control problems like (P1) and (P2) with nonstandard objective functions have not received much attention in the area of control theory. In contrast, their static analogues have been studied extensively during the last three decades. Recent surveys of fractional programming are given in $[8,18]$, and a fairly extensive bibliography is included in [18]. Similarly, a detailed account of discrete (and continuous) minmax theory and methods is available in [9].

Optimization problems involving norms occur in many areas of the decision sciences, applied mathematics, and engineering. These problems are encountered most frequently in location theory, approximation theory, and engineering design. A number of references dealing with various aspects of these problems are given in [26].

\section{Preliminaries}

In our discussion of optimality conditions for $(\mathrm{P})$ in the next section, we shall need an optimality result of [6] for the problem

$$
\text { (P4) Minimize } \int_{a}^{b}\left[f(x(t), u(t), t)+\|C(t) x(t)\|_{c}+\|E(t) u(t)\|_{e}\right] d t
$$

subject to (1)-(4) and

$$
\int_{a}^{b}\left[F_{k}(x(t), u(t), t)+\left\|I_{k}(t) x(t)\right\|_{c(k)}+\left\|J_{k}(t) u(t)\right\|_{d(k)}\right] d t \leq 0, \quad k \in \underline{\nu},
$$

where $f$ and $F_{k}, k \in \underline{\nu}$, are continuously differentiable real-valued functions defined on $\mathbb{R}^{n} \times \mathbb{R}^{m} \times[a, b] ; C(t), E(t), I_{k}(t)$, and $J_{k}(t), k \in \underline{\nu}$, are, respectively, $n_{1} \times n, m_{1} \times m, c_{k} \times n$, and $d_{k} \times m$ matrices whose entries are 
continuous real-valued functions defined on $[a, b]$, and $\|\cdot\|_{c},\|\cdot\|_{e},\|\cdot\|_{c(k)}$, and $\|\cdot\|_{d(k)}, k \in \underline{\nu}$, are arbitrary norms.

Although this problem is not explicitly considered in [6] in the exact form stated above, the method employed in $[4,5,6]$ can easily be modified and adapted for this problem. This would lead to the following optimality result for (P4).

Theorem 2.1. [6] Assume that the functions $f(., ., t), h_{j}(., ., t), j \in \underline{s}$, and $F_{k}(., ., t, k) \in \underline{\nu}$, are convex on $\mathbb{R}^{n} \times \mathbb{R}^{m}$ throughout $[a, b]$, that the Fréchet derivative of the linear map $(x, u) \rightarrow D x-A(t) x-B(t) u$ is surjective at a feasible solution $\left(x^{*}, u^{*}\right)$ of $(\mathrm{P} 4)$, and that the constraints of (P4) satisfy Slater's constraint qualification, that is, there exists $(\tilde{x}, \tilde{u}) \in C^{n}[a, b] \times P W S^{m}[a, b]$ such that $\tilde{x}(a)=\tilde{x}(b)=0$,

$$
\begin{gathered}
D \tilde{x}=A(t) \tilde{x}+B(t) \tilde{u}, \quad t \in[a, b], \\
h_{j}(\tilde{x}, \tilde{u}, t)+\left\|P_{j}(t) \tilde{x}\right\|_{p(j)}+\left\|Q_{j}(t) \tilde{u}\right\|_{q(j)}<0, \quad j \in \underline{s}, \\
\int_{a}^{b}\left[F_{k}(\tilde{x}, \tilde{u}, t)+\left\|I_{k}(t) \tilde{x}\right\|_{c(k)}+\left\|J_{k}(t) \tilde{u}\right\|_{d(k)}\right] d t<0, \quad k \in \underline{\nu},
\end{gathered}
$$

for all $t \in[a, b]$. Then $\left(x^{*}, u^{*}\right)$ is an optimal solution of (P4) if and only if there exist $v \in P W S^{n}[a, b], w \in P W S_{+}^{s}[a, b], z \in \mathbb{R}_{+}^{\nu}, \alpha \in$ $P W S^{n_{1}}[a, b], \beta \in P W S^{m_{1}}, \zeta^{j} \in P W S^{p_{j}}[a, b], \eta^{j} \in P W S^{q_{j}}[a, b], j \in$ $\underline{s} ; \theta^{k} \in P W S^{c_{k}}[a, b], \xi^{k} \in P W S^{d_{k}}[a, b], k \in \underline{\nu}$, such that the following relations hold for all $t \in[a, b]$ :

$$
\begin{aligned}
& \nabla_{1} f\left(x^{*}, u^{*}, t\right)+C(t)^{T} \alpha(t)+A(t)^{T} v(t) \\
&+\sum_{j=1}^{s} w_{j}(t)\left[\nabla_{1} h_{j}\left(x^{*}, u^{*}, t\right)+P_{j}(t)^{T} \zeta^{j}(t)\right]+ \\
& \sum_{k=1}^{\nu} z_{k}\left[\nabla_{1} F_{k}\left(x^{*}, u^{*}, t\right)+I_{k}(t)^{T} \theta^{k}(t)\right]+D v(t)=0, \\
& \nabla_{2} f\left(x^{*}, u^{*}, t\right)+E(t)^{T} \beta(t)+B(t)^{T} v(t) \\
&+\sum_{j=1}^{s} w_{j}(t)\left[\nabla_{2} h_{j}\left(x^{*}, u^{*}, t\right)+Q_{j}(t)^{T} \eta^{j}(t)\right]+ \\
& \sum_{k=1}^{\nu} z_{k}\left[\nabla_{2} F_{k}\left(x^{*}, u^{*}, t\right)+J_{k}(t)^{T} \xi^{k}(t)\right]=0,
\end{aligned}
$$




$$
\begin{aligned}
& \sum_{j=1}^{s} w_{j}(t)\left[h_{j}\left(x^{*}, u^{*}, t\right)+\left\|P_{j}(t) x^{*}\right\|_{p(j)}+\left\|Q_{j}(t) u^{*}\right\|_{q(j)}\right]=0 \\
& \int_{a}^{b} \sum_{k=1}^{\nu} z_{k}\left[F_{k}\left(x^{*}, u^{*}, t\right)+\left\|I_{k}(t) x^{*}\right\|_{c(k)}+\left\|J_{k}(t) u^{*}\right\|_{d(k)}\right] d t=0 \\
& \|\alpha(t)\|_{c}^{*} \leq 1, \quad\|\beta(t)\|_{e}^{*} \leq 1 \\
& \left\|\zeta^{j}(t)\right\|_{p(j)}^{*} \leq 1, \quad\left\|\eta^{j}(t)\right\|_{q(j)}^{*} \leq 1, j \in \underline{s} \\
& \left\|\theta^{k}(t)\right\|_{c(k)}^{*} \leq 1,\left\|\xi^{k}(t)\right\|_{d(k)}^{*} \leq 1, k \in \underline{\nu}, \\
& \alpha(t)^{T} C(t) x^{*}=\left\|C(t) x^{*}\right\|_{c}, \beta(t)^{T} E(t) u^{*}=\left\|E(t) u^{*}\right\|_{e}, \\
& \zeta^{j}(t)^{T} P_{j}(t) x^{*}=\left\|P_{j}(t) x^{*}\right\|_{p(j)}, \eta^{j}(t)^{T} Q_{j}(t) u^{*}=\left\|Q_{j}(t) u^{*}\right\|_{q(j)}, j \in \underline{s}, \\
& \theta^{k}(t)^{T} I_{k}(t) x^{*}=\left\|I_{k}(t) x^{*}\right\|_{c(k)}, \quad \xi^{k}(t)^{T} J_{k}(t) u^{*}=\left\|J_{k}(t) u^{*}\right\|_{d(k)}, k \in \underline{\nu}, \\
& \text { where } P W S_{+}^{s}[a, b]=\left\{w \in P W S^{s}[a, b]: w(t) \geq 0 \text { for all } t \in[a, b]\right\}, \mathbb{R}_{+}^{\nu} \\
& =\left\{z \in \mathbb{R}^{\nu}: z \geq 0\right\}, \nabla_{1} \psi \text { and } \nabla_{2} \psi \text { denote the partial gradients of the }
\end{aligned}
$$

In the above theorem, the argument $t$ of the vector-valued functions $\tilde{x}, \tilde{u}, x^{*}$, and $u^{*}$ was omitted for the sake of notational simplicity. This practice will be continued throughout the sequel.

\section{Optimality conditions}

Using a Dinkelbach-type [10] indirect approach based on Theorem 2.1 and a nonfractional equivalent problem, we first establish in this section a set of necessary and sufficient optimality conditions for $(\mathrm{P})$, and then show, with the aid of a second equivalent problem, that they are, in fact, also sufficient for optimality of a feasible solution of $(\mathrm{P})$. The auxiliary problem making this approach possible has the following form :

$$
\begin{aligned}
(P \mu) & \underset{(x, u) \in \mathbb{F}}{\operatorname{Minimize}} \max _{1 \leq i \leq r} \int_{a}^{b}\left\{f_{i}(x, u, t)\right. \\
+\left\|K_{i}(t) x\right\|_{k(i)}+\left\|L_{i}(t) u\right\|_{\ell(i)}-\mu\left[g_{i}(x, u, t)\right. & -M_{i}(t) x \|_{m(i)} \\
& \left.\left.-\left\|N_{i}(t) u\right\|_{n(i)}\right]\right\} d t
\end{aligned}
$$

where $\mu \in \mathbb{R}_{+} \equiv[0, \infty)$ is a parameter. 
This problem can be utilized to investigate certain aspects of $(\mathrm{P})$. The relationship between $(\mathrm{P})$ and $(\mathrm{P} \mu)$ needed for our present purposes is stated in the following lemma whose proof is straightforward and hence omitted.

Lemma 3.1. Let $\mu^{*}$ be the optimal value of $(P)$ and let $v(\mu)$ be the optimal value of $(P \mu)$ for any $\mu \in \mathbb{R}_{+}$such that $(P \mu)$ has an optimal solution. If $\left(x^{*}, u^{*}\right)$ is an optimal solution of $(P)$, then $\left(x^{*}, u^{*}\right)$ is an optimal solution of $\left(\mathrm{P} \mu^{*}\right)$ and $v\left(\mu^{*}\right)=0$.

It is clear that $(\mathrm{P} \mu)$ is equivalent to the following problem : $(\mathrm{EP} \mu)$ Minimize $\xi$ subject to $(x, u) \in \mathbb{F}, \xi \in \mathbb{R}$, and

$$
\begin{array}{r}
\int_{a}^{b}\left\{f_{i}(x, u, t)+\left\|K_{i}(t) x\right\|_{k(i)}+\left\|L_{i}(t) u\right\|_{\ell(i)}-\mu\left[g_{i}(x, u, t)-\left\|M_{i}(t) x\right\|_{m(i)}\right.\right. \\
\left.\left.-\left\|N_{i}(t) u\right\|_{n(i)}\right]\right\} d t \leq \xi, \quad i \in \underline{r} .
\end{array}
$$

In view of Lemma 3.1 and the equivalence between $(\mathrm{P} \mu)$ and $(\mathrm{EP} \mu)$, it is clear that if $\left(x^{*}, u^{*}\right)$ is an optimal solution of $(\mathrm{P})$ with optimal value $\mu^{*}$, then $\left(x^{*}, u^{*}, \xi^{*}\right)=\left(x^{*}, u^{*}, 0\right)$ is an optimal solution of $\left(\operatorname{EP} \mu^{*}\right)$. We shall make use of this observation in the proof of Theorem 3.1 which contains the main result of this section. Prior to stating this theorem, however, we specify our basic assumptions which will remain in force throughout the sequel.

(a) The functions $f_{i}(., ., t),-g_{i}(., ., t), i \in \underline{r}$, and $h_{j}(., ., t), j \in \underline{s}$, are convex on $\mathbb{R}^{n} \times \mathbb{R}^{m}$ throughout $[a, b]$.

(b) The constraints of $(\mathrm{P})$ satisfy Slater's constraint qualification (see Theorem 2.1).

Despite the above convexity assumptions, $(\mathrm{P})$ is not a convex problem because of the fractional nature of its objective function. However, this difficulty can be circumvented by resorting to the intermediate problems $(\mathrm{P} \mu)$ and $(\mathrm{EP} \mu)$ which are convex problems by virtue of our assumptions specified in (a) above and in the description of $(\mathrm{P})$.

THEOREM 3.1. Let $\left(x^{*}, u^{*}\right)$ be an optimal solution of $(P)$. Then there exist $\lambda^{*} \in \mathbb{R}_{+}^{r}, \sum_{i=1}^{r} \lambda_{i}^{*}=1, \mu^{*} \in \mathbb{R}_{+}, v^{*} \in P W S^{n}[a, b], w^{*} \in$ $P W S_{+}^{s}[a, b], \alpha^{* i} \in P W S^{k_{i}}[a, b], \beta^{* i} \in P W S^{\ell_{i}}[a, b], \gamma^{* i} \in P W S^{m_{i}}[a, b]$, $\delta^{* i} \in P W S^{n_{i}}[a, b], i \in \underline{r}, \zeta^{* j} \in P W S^{p_{j}}[a, b], \eta^{* j} \in P W S^{q_{j}}[a, b], j \in \underline{s}$, 
such that the following relations hold for all $t \in[a, b]$ :

(5)

$$
\begin{aligned}
\sum_{i=1}^{r} \lambda_{i}^{*}\{ & \nabla_{1} f_{i}\left(x^{*}, u^{*}, t\right)+K_{i}(t)^{T} \alpha^{* i}(t) \\
- & \left.\mu^{*}\left[\nabla_{1} g_{i}\left(x^{*}, u^{*}, t\right)-M_{i}(t)^{T} \gamma^{* i}(t)\right]\right\}+A(t)^{T} v^{*}(t) \\
& +\sum_{j=1}^{s} w_{j}^{*}(t)\left[\nabla_{1} h_{j}\left(x^{*}, u^{*}, t\right)+P_{j}(t)^{T} \zeta^{* j}(t)\right]+D v^{*}(t)=0
\end{aligned}
$$

(6) $\sum_{i=1}^{r} \lambda_{i}^{*}\left\{\nabla_{2} f_{i}\left(x^{*}, u^{*}, t\right)+L_{i}(t)^{T} \beta^{* i}(t)\right.$

$$
\begin{aligned}
& \left.-\mu^{*}\left[\nabla_{2} g_{i}\left(x^{*}, u^{*}, t\right)-N_{i}(t)^{T} \delta^{* i}(t)\right]\right\}+B(t)^{T} v^{*}(t) \\
& +\sum_{j=1}^{s} w_{j}^{*}(t)\left[\nabla_{2} h_{j}\left(x^{*}, u^{*}, t\right)+Q_{j}(t)^{T} \eta^{* j}(t)\right]=0,
\end{aligned}
$$

(7)

$$
\sum_{j=1}^{s} w_{j}^{*}(t)\left[h_{j}\left(x^{*}, u^{*}, t\right)+\left\|P_{j}(t) x^{*}\right\|_{p(j)}+\left\|Q_{j}(t) u^{*}\right\|_{q(j)}\right]=0
$$

(8) $\int_{a}^{b} \sum_{i=1}^{r} \lambda_{i}^{*}\left\{f_{i}\left(x^{*}, u^{*}, t\right)+\left\|K_{i}(t) x^{*}\right\|_{k(i)}+\left\|L_{i}(t) u^{*}\right\|_{\ell(i)}\right.$

$$
\begin{array}{r}
-\mu^{*}\left[g_{i}\left(x^{*}, u^{*}, t\right)-\left\|M_{i}(t) x^{*}\right\|_{m(i)}\right. \\
\left.\left.-\left\|N_{i}(t) u^{*}\right\|_{n(i)}\right]\right\} d t=0,
\end{array}
$$

(9) $\left\|\alpha^{* i}(t)\right\|_{k(i)}^{*} \leq 1,\left\|\beta^{* i}(t)\right\|_{\ell(i)}^{*} \leq 1$,

$$
\left\|\gamma^{* i}(t)\right\|_{m(i)}^{*} \leq 1, \quad\left\|\delta^{* i}(t)\right\|_{n(i)}^{*} \leq 1, \quad i \in \underline{r},
$$

$$
\left\|\zeta^{* j}(t)\right\|_{p(j)}^{*} \leq 1,\left\|\eta^{* j}(t)\right\|_{q(j)}^{*} \leq 1, \quad j \in \underline{s},
$$

(11) $\alpha^{* i}(t)^{T} K_{i}(t) x^{*}=\left\|K_{i}(t) x^{*}\right\|_{k(i)}, \quad \beta^{* i}(t)^{T} L_{i}(t) u^{*}=\left\|L_{i}(t) u^{*}\right\|_{\ell(i)}$, $\gamma^{* i}(t)^{T} M_{i}(t) x^{*}=\left\|M_{i}(t) x^{*}\right\|_{m(i)}, \delta^{* i}(t)^{T} N_{i}(t) u^{*}=\left\|N_{i}(t) u^{*}\right\|_{n(i)}, i \in \underline{r}$,

$$
\zeta^{* j}(t)^{T} P_{j}(t) x^{*}=\left\|P_{j}(t) x^{*}\right\|_{p(j)}, \eta^{* j}(t)^{T} Q_{j}(t) u^{*}=\left\|Q_{j}(t) u^{*}\right\|_{q(j)}, j \in \underline{s} .
$$


Proof. Since $\left(x^{*}, u^{*}\right)$ is an optimal solution of $(\mathrm{P})$, by Lemma 3.1, it is an optimal solution of $\left(\mathrm{P} \mu^{*}\right)$, where $\mu^{*}$ is the optimal value of (P). This implies that $\left(x^{*}, u^{*}, \xi^{*}\right)=\left(x^{*}, u^{*}, 0\right)$ is an optimal solution of $\left(\mathrm{EP} \mu^{*}\right)$. By hypothesis, there exists $(\tilde{x}, \tilde{u}) \in C^{n}[a, b] \times P W S^{m}[a, b]$, with $\tilde{x}(a)=\tilde{x}(b)=0$, at which Slater's constraint qualification holds for $(\mathrm{P})$. Because of the special structure of the constraints of $\left(\mathrm{EP} \mu^{*}\right)$, it is obvious that for some $\tilde{\xi} \in \mathbb{R}$, Slater's constraint qualification holds for $\left(\mathrm{EP} \mu^{*}\right)$ at $(\tilde{x}, \tilde{u}, \tilde{\xi})$. Therefore, by Theorem 2.1 (applied to $\left(\mathrm{EP} \mu^{*}\right)$ ), there exist $\lambda^{*}, \mu^{*}, v^{*}, w^{*}, \alpha^{* i}, \beta^{* i}, \gamma^{* i}, \delta^{* i}, i \in \underline{r}, \zeta^{* j}$, and $\eta^{* j}, j \in \underline{s}$, as specified above, such that (5) -(12) hold for all $t \in[a, b]$.

We next show that the necessary optimality conditions of Theorem 3.1 are also sufficient for optimality of $\left(x^{*}, u^{*}\right)$. For this we need two simple lemmas. The first lemma is a well-known result, called the generalized Cauchy-Schwarz inequality, whereas the second lemma provides an alternative expression for the objective function of $(\mathrm{P})$ whose proof is straightforward and hence omitted.

Lemma 3.2. [12] For any $y, z \in \mathbb{R}^{\nu}$, one has $y^{T} z \leq\|y\|\|z\|^{*}$.

Lemma 3.3. For each $(x, u) \in C^{n}[a, b] \times P W S^{m}[a, b]$, one has

$$
\begin{aligned}
\varphi(x, u) & \equiv \max _{1 \leq i \leq r} \frac{\int_{a}^{b}\left[f_{i}(x, u, t)+\left\|K_{i}(t) x\right\|_{k(i)}+\left\|L_{i}(t) u\right\|_{\ell(i)}\right] d t}{\int_{a}^{b}\left[g_{i}(x, u, t)-\left\|M_{i}(t) x\right\|_{m(i)}-\left\|N_{i}(t) u\right\|_{n(i)}\right] d t} \\
& =\max _{\lambda \in \Lambda} \frac{\int_{a}^{b} \sum_{i=1}^{r} \lambda_{i}\left[f_{i}(x, u, t)+\left\|K_{i}(t) x\right\|_{k(i)}+\left\|L_{i}(t) u\right\|_{\ell(i)}\right] d t}{\int_{a}^{b} \sum_{i=1}^{r} \lambda_{i}\left[g_{i}(x, u, t)-\left\|M_{i}(t) x\right\|_{m(i)}-\left\|N_{i}(t) u\right\|_{n(i)}\right] d t}
\end{aligned}
$$

where

$$
\Lambda=\left\{\lambda \in \mathbb{R}_{+}^{r}: \sum_{i=1}^{r} \lambda_{i}=1\right\}
$$

THEOREM 3.2. Let $\left(x^{*}, u^{*}\right) \in \mathbb{F}$, let $\mu^{*}=\varphi\left(x^{*}, u^{*}\right)$, and assume that there exist $\lambda^{*} \in \Lambda, \mu^{*} \in \mathbb{R}_{+}, v^{*} \in P W S^{n}[a, b], w^{*} \in P W S_{+}^{s}[a, b], \alpha^{* i} \in$ $P W S^{k_{i}}[a, b], \beta^{* i} \in P W S^{\ell_{i}}[a, b], \gamma^{* i} \in P W S^{m_{i}}[a, b], \delta^{* i} \in P W S^{n_{i}}[a, b]$, $i \in \underline{r}, \zeta^{* j} \in P W S^{p_{j}}[a, b], \eta^{* j} \in P W S^{q_{j}}[a, b], j \in \underline{s}$, such that (5)-(12) hold for all $t \in[a, b]$. Then $\left(x^{*}, u^{*}\right)$ is an optimal solution of $(P)$. 
Proof. Let $(x, u)$ be an arbitrary feasible solution of $(\mathrm{P})$. Keeping in mind that $\lambda^{*} \geq 0, \mu^{*} \geq 0$, and $w^{*}(t) \geq 0$ for all $t \in[a, b]$, we have

$$
\begin{aligned}
& \left.\int_{a}^{b} \sum_{i=1}^{r} \lambda_{i}^{*}\left\{f_{i}(x, u, t)+\| K_{i}(t) x\right)\left\|_{k(i)}+\right\| L_{i}(t) u \|_{\ell(i)}\right] \\
& \left.-\mu^{*}\left[g_{i}(x, u, t)-\left\|M_{i}(t) x\right\|_{m(i)}-\left\|N_{i}(t) u\right\|_{n(i)}\right]\right\} d t \\
= & \int_{a}^{b} \sum_{i=1}^{r} \lambda_{i}^{*}\left\{f_{i}(x, u, t)+\left\|K_{i}(t) x\right\|_{k(i)}+\left\|L_{i}(t) u\right\|_{\ell(i)}\right. \\
& -\mu^{*}\left[g_{i}(x, u, t)-\left\|M_{i}(t) x\right\|_{m(i)}-\left\|N_{i}(t) u\right\|_{n(i)}\right] \\
& -f_{i}\left(x^{*}, u^{*}, t\right)-\alpha^{* i}(t)^{T} K_{i}(t) x^{*}-\beta^{* i}(t)^{T} L_{i}(t) u^{*} \\
& +\mu^{*}\left[g_{i}\left(x^{*}, u^{*}, t\right)-\gamma^{* i}(t)^{T} M_{i}(t) x^{*}\right. \\
& \left.\left.-\delta^{* i}(t)^{T} N_{i}(t) u^{*}\right]\right\} d t(\mathrm{by}(8) \text { and }(11)) \\
\geq & \int_{a}^{b} \sum_{i=1}^{r} \lambda_{i}^{*}\left\{\nabla_{1} f_{i}\left(x^{*}, u^{*}, t\right)^{T}\left(x-x^{*}\right)+\nabla_{2} f_{i}\left(x^{*}, u^{*}, t\right)^{T}\left(u-u^{*}\right)\right. \\
& -\mu^{*}\left[\nabla_{1} g_{i}\left(x^{*}, u^{*}, t\right)^{T}\left(x-x^{*}\right)+\nabla_{2} g_{i}\left(x^{*}, u^{*}, t\right)^{T}\left(u-u^{*}\right)\right] \\
& \left.+\left\|K_{i}(t) x\right\|_{k(i)}+\left\|L_{i}(t) u\right\|_{\ell(i)}+\mu^{*}\left\|M_{i}(t) x\right\|_{m(i)}+\mu^{*}\left\|N_{i}(t) u\right\|_{n(i)}\right] \\
& -\alpha^{* i}(t)^{T} K_{i}(t) x^{*}-\beta^{* i}(t)^{T} L_{i}(t) u^{*}-\mu^{*} \gamma^{* i}(t)^{T} M_{i}(t) x^{*} \\
& \left.\left.-\mu^{*} \delta^{* i}(t)^{T} N_{i}(t) u^{*}\right]\right\} d t \\
& (b y t h e c o n v e x i t y
\end{aligned}
$$

(by the convexity of $f_{i}(., ., t)$ and $-g_{i}(., ., t), i \in \underline{r}$ )

$$
\begin{aligned}
= & \int_{a}^{b}\left\{\sum _ { i = 1 } ^ { r } \lambda _ { i } ^ { * } \left\{\left[-\alpha^{* i}(t)^{T} K_{i}(t)-\mu^{*} \gamma^{* i}(t)^{T} M_{i}(t)\right]\left(x-x^{*}\right)\right.\right. \\
& +\left[-\beta^{* i}(t)^{T} L_{i}(t)-\mu^{*} \delta^{* i}(t)^{T} N_{i}(t)\right]\left(u-u^{*}\right)+\left\|K_{i}(t) x(t)\right\|_{k(i)} \\
& \left.+\left\|L_{i}(t) u\right\|_{\ell(i)}+\mu^{*}\left\|M_{i}(t) x\right\|_{m(i)}+\mu^{*}\left\|N_{i}(t) u\right\|_{n(i)}\right] \\
& -\alpha^{* i}(t)^{T} K_{i}(t) x^{*}-\beta^{* i}(t)^{T} L_{i}(t) u^{*}-\mu^{*} \gamma^{* i}(t)^{T} M_{i}(t) x^{*} \\
& \left.-\mu^{*} \delta^{* i}(t)^{T} N_{i}(t) u^{*}\right\}-\left\{v^{*}(t)^{T} A(t)+\sum_{j=1}^{s} w_{j}^{*}(t)\left[\nabla_{1} h_{j}\left(x^{*}, u^{*}, t\right)^{T}\right.\right. \\
& \left.+\zeta^{* j}(t)^{T} P_{j}(t)+D v^{*}(t)^{T}\right\}\left(x-x^{*}\right) \\
& -\left\{v^{*}(t)^{T} B(t)+\sum_{j=1}^{s} w_{j}^{*}(t)\left[\nabla_{2} h_{j}\left(x^{*}, u^{*}, t\right)^{T}\right.\right.
\end{aligned}
$$




$$
\begin{aligned}
& \left.\left.\left.\left.+\eta^{* j}(t)^{T} Q_{j}(t)\right]\right\}\left(u-u^{*}\right)\right\} d t \text { (by (5) and }(6)\right) \\
\geq & \int_{a}^{b}\left\{\sum _ { i = 1 } ^ { r } \lambda _ { i } ^ { * } \left[-\left\|K_{i}(t) x\right\|_{k(i)}\left\|\alpha^{* i}(t)\right\|_{k(i)}^{*}-\mu^{*}\left\|M_{i}(t) x\right\|_{m(i)}\left\|\gamma^{* i}(t)\right\|_{m(i)}^{*}\right.\right. \\
& -\left\|L_{i}(t) u\right\|_{\ell(i)}\left\|\beta^{* i}(t)\right\|_{\ell(i)}^{*}-\mu^{*}\left\|N_{i}(t) u\right\|_{n(i)}\left\|\delta^{* i}(t)\right\|_{n(i)}^{*}+\left\|K_{i}(t) x\right\|_{k(i)} \\
& \left.+\left\|L_{i}(t) u\right\|_{\ell(i)}+\mu^{*}\left\|M_{i}(t) x\right\|_{m(i)}+\mu^{*}\left\|N_{i}(t) u\right\|_{n(i)}\right] \\
& +v^{*}(t)^{T}\left[D x-D x^{*}-A(t)\left(x-x^{*}\right)-B(t)\left(u-u^{*}\right)\right] \\
& -\sum_{j=1}^{s} w_{j}^{*}(t)\left[\left\|P_{j}(t) x\right\|_{p(j)} \zeta^{* j}(t)\left\|_{p(j)}^{*}+\right\| Q_{j}(t) u\left\|_{q(j)}\right\| \eta^{* j}(t) \|_{q(j)}^{*}\right] \\
& +\sum_{j=1}^{s} w_{j}^{*}(t)\left[\zeta^{* j}(t)^{T} P_{j}(t) x^{*}+\eta^{* j}(t)^{T} Q_{j}(t) u^{*}\right] \\
& \left.+\sum_{j=1}^{s} w_{j}^{*}(t)\left[h_{j}\left(x^{*}, u^{*}, t\right)-h_{j}(x, u, t)\right]\right\} d t \text { (by Lemma 3.2, integration }
\end{aligned}
$$

by parts, and convexity of $\left.h_{j}(., ., t), j \in \underline{s}\right)$

$$
\begin{aligned}
\geq & \int_{a}^{b}\left\{v^{*}(t)^{T}[D x-A(t) x-B(t) u]-v^{*}(t)^{T}\left[D x^{*}-A(t) x^{*}-B(t) u^{*}\right]\right. \\
& +\sum_{j=1}^{s} w_{j}^{*}(t)\left[h_{j}\left(x^{*}, u^{*}, t\right)+\zeta^{* j}(t)^{T} P_{j}(t) x^{*}+\eta^{* j}(t)^{T} Q_{j}(t) u^{*}\right] \\
& \left.-\sum_{j=1}^{s} w_{j}^{*}(t)\left[h_{j}(x, u, t)+\left\|P_{j}(t) x\right\|_{p(j)}+\left\|Q_{j}(t) u\right\|_{q(j)}\right]\right\} d t
\end{aligned}
$$

(by (9) and (10))

$\geq 0$ (by (7), (12), and feasibility of $\left(x^{*}, u^{*}\right)$ and $\left.(x, u)\right)$.

Now using this inequality and Lemma 3.3, we see that

$$
\begin{aligned}
\varphi(x, u) & =\max _{\lambda \in \Lambda} \frac{\int_{a}^{b} \sum_{i=1}^{r} \lambda_{i}\left[f_{i}(x, u, t)+\left\|K_{i}(t) x\right\|_{k(i)}+\left\|L_{i}(t) u\right\|_{\ell(i)}\right] d t}{\int_{a}^{b} \sum_{i=1}^{r} \lambda_{i}\left[g_{i}(x, u, t)-\left\|M_{i}(t) x\right\|_{m(i)}-\left\|N_{i}(t) u\right\|_{n(i)}\right] d t} \\
& \geq \frac{\int_{a}^{b} \sum_{i=1}^{r} \lambda_{i}^{*}\left[f_{i}(x, u, t)+\left\|K_{i}(t) x\right\|_{k(i)}+\left\|L_{i}(t) u\right\|_{\ell(i)}\right] d t}{\int_{a}^{b} \sum_{i=1}^{r} \lambda_{i}^{*}\left[g_{i}(x, u, t)-\left\|M_{i}(t) x\right\|_{m(i)}-\left\|N_{i}(t) u\right\|_{n(i)}\right] d t} \\
& \geq \mu^{*}=\varphi\left(x^{*}, u^{*}\right) .
\end{aligned}
$$

Since $(x, u)$ was an arbitrary feasible solution of $(\mathrm{P})$, we conclude from the above inequality that $\left(x^{*}, u^{*}\right)$ is an optimal solution of $(\mathrm{P})$. 
A review of the steps of the above proof will readily show that the following slightly modified version of Theorem 3.2 is also valid.

Theorem 3.3. Assume as in Theorem 3.2 except that (5) and (6) are replaced by the following inequalities:

$$
\begin{aligned}
&\left\{\sum _ { i = 1 } ^ { r } \lambda _ { i } ^ { * } \left\{\nabla_{1} f_{i}\left(x^{*}, u^{*}, t\right)^{T}+\right.\right. \alpha^{* i}(t)^{T} K_{i}(t)-\mu^{*}\left[\nabla_{1} g_{i}\left(x^{*}, u^{*}, t\right)^{T}\right. \\
&\left.\left.-\gamma^{* i}(t)^{T} M_{i}(t)\right]\right\}+v^{*}(t)^{T} A(t)+\sum_{j=1}^{s} w_{j}^{*}(t)\left[\nabla_{1} h_{j}\left(x^{*}, u^{*}, t\right)^{T}\right. \\
&\left.\left.+\zeta^{* j}(t)^{T} P_{j}(t)\right]+D v^{*}(t)^{T}\right\}\left(x-x^{*}\right) \geq 0
\end{aligned}
$$

for all $t \in[a, b]$ and $x \in C^{n}[a, b]$ such that $(x, u) \in \mathbb{F}$ for some $u \in$ $P W S^{m}[a, b]$,

$$
\begin{aligned}
\sum_{i=1}^{r} \lambda_{i}^{*}\left\{\nabla_{2}\right. & f_{i}\left(x^{*}, u^{*}, t\right)^{T}+\beta^{* i}(t)^{T} L_{i}(t) \\
& \left.-\mu^{*}\left[\nabla_{2} g_{i}\left(x^{*}, u^{*}, t\right)^{T}-\delta^{* i}(t)^{T} N_{i}(t)\right]\right\}+v^{*}(t)^{T} B(t) \\
& \left.+\sum_{j=1}^{s} w_{j}^{*}(t)\left[\nabla_{2} h_{j}\left(x^{*}, u^{*}, t\right)^{T}+\eta^{* j}(t)^{T} Q_{j}(t)\right]\right\}\left(u-u^{*}\right) \geq 0
\end{aligned}
$$

for all $t \in[a, b]$ and $u \in P W S^{m}[a, b]$ such that $(x, u) \in \mathbb{F}$ for some $x \in C^{n}[a, b]$. Then $\left(x^{*}, u^{*}\right)$ is an optimal solution of $(P)$.

In the next section, we shall see that the contents of Theorems 3.1 -3.3 provide clear guidelines for developing a parametric duality theory for $(P)$. In particular, the two versions of the sufficiency criteria can be utilized to construct two dual problems for $(\mathrm{P})$. We shall elaborate on the differences between Theorems 3.2 and 3.3 later in the context of these duality formulations.

The optimality conditions stated in Theorems 3.1-3.3 contain the parameter $\mu^{*}$ which was introduced as a result of our indirect approach via the auxiliary problem $(\mathrm{P} \mu)$. However, from the form of these optimality conditions it is evident that this parameter can readily be eliminated. Indeed, this can be done by simply solving for $\mu^{*}$ in (8), substituting the result in (5) and (6), simplifying, and redefining the multiplier vectors $v^{*}(t)$ and $w^{*}(t)$. This process leads to the following parameter-free versions of Theorems 3.1-3.3. 
THEOREM 3.4. A feasible solution $\left(x^{\circ}, u^{\circ}\right)$ of $(P)$ is optimal if and only if there exist $\lambda^{\circ} \in \Lambda, v^{\circ} \in P W S^{n}[a, b], w^{\circ} \in P W S_{+}^{s}[a, b], \alpha^{\circ i} \in$ $P W S^{k_{i}}[a, b], \beta^{\circ i} \in P W S^{\ell_{i}}[a, b], \gamma^{\circ i} \in P W S^{m_{i}}[a, b], \delta^{\circ i} \in P W S^{n_{i}}[a, b]$, $i \in \underline{r}, \zeta^{\circ j} \in P W S^{p_{j}}[a, b], \eta^{\circ j} \in P W S^{q_{j}}[a, b], j \in \underline{s}$, such that the following relations hold for all $t \in[a, b]$ :

$$
\begin{aligned}
& \sum_{i=1}^{r} \lambda_{i}^{\circ}\left\{\Psi\left(x^{\circ}, u^{\circ}, \lambda^{\circ}\right)\left[\nabla_{1} f_{i}\left(x^{\circ}, u^{\circ}, t\right)+K_{i}(t)^{T} \alpha^{\circ i}(t)\right]\right. \\
& \left.-\Phi\left(x^{\circ}, u^{\circ}, \lambda^{\circ}\right)\left[\nabla_{1} g_{i}\left(x^{\circ}, u^{\circ}, t\right)-M_{i}(t)^{T} \gamma^{\circ i}(t)\right]\right\}+A(t)^{T} v^{\circ}(t) \\
& +\sum_{j=1}^{s} w_{j}^{\circ}(t)\left[\nabla_{1} h_{j}\left(x^{\circ}, u^{\circ}, t\right)+P_{j}(t)^{T} \zeta^{\circ j}(t)\right]+D v^{\circ}(t)=0, \\
& \sum_{i=1}^{r} \lambda_{i}^{\circ}\left\{\Psi\left(x^{\circ}, u^{\circ}, \lambda^{\circ}\right)\left[\nabla_{2} f_{i}\left(x^{\circ}, u^{\circ}, t\right)+L_{i}(t)^{T} \beta^{\circ i}(t)\right]\right. \\
& \left.-\Phi\left(x^{\circ}, u^{\circ}, \lambda^{\circ}\right)\left[\nabla_{2} g_{i}\left(x^{\circ}, u^{\circ}, t\right)-N_{i}(t)^{T} \delta^{\circ i}(t)\right]\right\} \\
& +B(t)^{T} v^{\circ}(t)+\sum_{j=1}^{s} w_{j}^{\circ}(t)\left[\nabla_{2} h_{j}\left(x^{\circ}, u^{\circ}, t\right)+Q_{j}(t)^{T} \eta^{\circ j}(t)\right]=0
\end{aligned}
$$

$$
\sum_{j=1}^{s} w_{j}^{\circ}(t)\left[h_{j}\left(x^{\circ}, u^{\circ}, t\right)+\left\|P_{j}(t) x^{\circ}\right\|_{p(j)}+\left\|Q_{j}(t) u^{\circ}\right\|_{q(j)}\right]=0
$$

$$
\varphi\left(x^{\circ}, u^{\circ}\right)=\frac{\Phi\left(x^{\circ}, u^{\circ}, \lambda^{\circ}\right)}{\Psi\left(x^{\circ}, u^{\circ}, \lambda^{\circ}\right)}
$$

(15) $\left\|\alpha^{\circ i}(t)\right\|_{k(i)}^{*} \leq 1,\left\|\beta^{\circ i}(t)\right\|_{\ell(i)}^{*} \leq 1$,

$$
\left\|\gamma^{\circ i}(t)\right\|_{m(i)}^{*} \leq 1, \quad\left\|\delta^{\circ i}(t)\right\|_{n(i)}^{*} \leq 1, \quad i \in \underline{r},
$$

$$
\left\|\zeta^{\circ j}(t)\right\|_{p(j)}^{*} \leq 1,\left\|\eta^{\circ j}(t)\right\|_{q(j)}^{*} \leq 1, \quad j \in \underline{s}
$$

(17) $\alpha^{\circ i}(t)^{T} K_{i}(t) x^{\circ}=\left\|K_{i}(t) x^{\circ}\right\|_{k(i)}, \beta^{\circ i}(t)^{T} L_{i}(t) u^{\circ}=\left\|L_{i}(t) u^{\circ}\right\|_{\ell(i)}$, $\gamma^{\circ i}(t)^{T} M_{i}(t) x^{\circ}=\left\|M_{i}(t) x^{\circ}\right\|_{m(i)}, \delta^{\circ i}(t)^{T} N_{i}(t) u^{\circ}=\left\|N_{i}(t) u^{\circ}\right\|_{n(i)}, i \in \underline{r}$,

$$
\zeta^{\circ j}(t)^{T} P_{j}(t) x^{\circ}=\left\|P_{j}(t) x^{\circ}\right\|_{p(j)}, \quad \eta^{\circ j}(t)^{T} Q_{j}(t) u^{\circ}=\left\|Q_{j}(t) u^{\circ}\right\|_{q(j)}, j \in \underline{s},
$$


where

$\Phi\left(x^{\circ}, u^{\circ}, \lambda^{\circ}\right)=\int_{a}^{b} \sum_{i=1}^{r} \lambda_{i}^{\circ}\left[f_{i}\left(x^{\circ}, u^{\circ}, t\right)+\left\|K_{i}(t) x^{\circ}\right\|_{k(i)}+\left\|L_{i}(t) u^{\circ}\right\|_{\ell(i)}\right] d t$

and

$\Psi\left(x^{\circ}, u^{\circ}, \lambda^{\circ}\right)=\int_{a}^{b} \sum_{i=1}^{r} \lambda_{i}^{\circ}\left[g_{i}\left(x^{\circ}, u^{\circ}, t\right)-\left\|M_{i}(t) x^{\circ}\right\|_{m(i)}-\left\|N_{i}(t) u^{\circ}\right\|_{n(i)}\right] d t$.

Theorem 3.5. A feasible solution $\left(x^{\circ}, u^{\circ}\right)$ of $(P)$ is optimal if and only if there exist $\lambda^{\circ}, v^{\circ}, w^{\circ}, \alpha^{\circ i}, \beta^{\circ i}, \gamma^{\circ i}, \delta^{\circ i}, i \in \underline{r}, \zeta^{\circ j}$, and $\eta^{\circ j}, j \in$ $\underline{s}$, as specified in Theorem 3.4, such that (13)-(18) and the following inequalities hold for all $t \in[a, b]$ :

$$
\begin{aligned}
& \left\{\sum _ { i = 1 } ^ { r } \lambda _ { i } ^ { \circ } \left\{\Psi\left(x^{\circ}, u^{\circ}, \lambda^{\circ}\right)\left[\nabla_{1} f_{i}\left(x^{\circ}, u^{\circ}, t\right)^{T}+\alpha^{\circ}(t)^{T} K_{i}(t)\right]\right.\right. \\
& \left.-\Phi\left(x^{\circ}, u^{\circ}, \lambda^{\circ}\right)\left[\nabla_{1} g_{i}\left(x^{\circ}, u^{\circ}, t\right)^{T}-\gamma^{\circ i}(t)^{T} M_{i}(t)\right]\right\} \\
& +v^{\circ}(t)^{T} A(t)+\sum_{j=1}^{s} w_{j}^{\circ}(t)\left[\nabla_{1} h_{j}\left(x^{\circ}, u^{\circ}, t\right)^{T}+\zeta^{\circ j}(t)^{T} P_{j}(t)\right] \\
& \left.+D v^{\circ}(t)^{T}\right\}\left(x-x^{\circ}\right) \geq 0
\end{aligned}
$$

for all $t \in[a, b]$ and $x \in C^{n}[a, b]$ such that $(x, u) \in \mathbb{F}$ for some $u \in$ $P W S^{m}[a, b]$,

$$
\begin{aligned}
& \left\{\sum _ { i = 1 } ^ { r } \lambda _ { i } ^ { \circ } \left\{\Psi\left(x^{\circ}, u^{\circ}, \lambda^{\circ}\right)\left[\nabla_{2} f_{i}\left(x^{\circ}, u^{\circ}, t\right)^{T}+\beta^{\circ i}(t)^{T} L_{i}(t)\right]\right.\right. \\
& \left.-\Phi\left(x^{\circ}, u^{\circ}, \lambda^{\circ}\right)\left[\nabla_{2} g_{i}\left(x^{\circ}, u^{\circ}, t\right)^{T}-\delta^{\circ i}(t)^{T} N_{i}(t)\right]\right\}+v^{\circ}(t)^{T} B(t) \\
& \left.+\sum_{j=1}^{s} w_{j}^{\circ}(t)\left[\nabla_{2} h_{j}\left(x^{\circ}, u^{\circ}, t\right)^{T}+\eta^{\circ j}(t)^{T} Q_{j}(t)\right]\right\}\left(u-u^{\circ}\right) \geq 0
\end{aligned}
$$

for all $t \in[a, b]$ and $u \in P W S^{m}[a, b]$ such that $(x, u) \in \mathbb{F}$ for some $x \in C^{n}[a, b]$.

Theorems 3.4 and 3.5 form the basis for constructing several parameter-free duality models for $(\mathrm{P})$. They will be discussed in Sections 5 and 6. 


\section{Duality model I}

In this section, we formulate two parametric dual problems for $(\mathrm{P})$ and establish weak, strong, and strict converse duality theorems. These problems, whose forms and features are motivated by Theorems 3.1-3.3, can be stated as follows:

(DI) Maximize $\mu$ subject to (1), (4), and

$$
\begin{gathered}
\sum_{i=1}^{r} \lambda_{i}\left\{\nabla_{1} f_{i}(x, u, t)+K_{i}(t)^{T} \alpha^{i}(t)-\mu\left[\nabla_{1} g_{i}(x, u, t)-M_{i}(t)^{T} \gamma^{i}(t)\right]\right\} \\
+A(t)^{T} v(t)+\sum_{j=1}^{s} w_{j}(t)\left[\nabla_{1} h_{j}(x, u, t)+P_{j}(t)^{T} \zeta^{j}(t)\right]+D v(t)=0, t \in[a, b],
\end{gathered}
$$

$$
\begin{aligned}
& \sum_{i=1}^{r} \lambda_{i}\left\{\nabla_{2} f_{i}(x, u, t)+L_{i}(t)^{T} \beta^{i}(t)-\mu\left[\nabla_{2} g_{i}(x, u, t)-N_{i}(t)^{T} \delta^{i}(t)\right]\right\} \\
& +B(t)^{T} v(t)+\sum_{j=1}^{s} w_{j}(t)\left[\nabla_{2} h_{j}(x, u, t)+Q_{j}(t)^{T} \eta^{j}(t)\right]=0, \quad t \in[a, b]
\end{aligned}
$$

$$
\begin{aligned}
\int_{a}^{b}\{ & \sum_{i=1}^{r} \lambda_{i}\left\{f_{i}(x, u, t)+\alpha^{i}(t)^{T} K_{i}(t) x+\beta^{i}(t)^{T} L_{i}(t) u-\mu\left[g_{i}(x, u, t)\right.\right. \\
- & \left.\left.\gamma^{i}(t)^{T} M_{i}(t) x-\delta(t)^{T} N_{i}(t) u\right]\right\}-v(t)^{T}[D x-A(t) x-B(t) u] \\
& \left.+\sum_{j=1}^{s} w_{j}(t)\left[h_{j}(x, u, t)+\zeta^{j}(t)^{T} P_{j}(t) x+\eta^{j}(t)^{T} Q_{j}(t) u\right]\right\} d t \geq 0
\end{aligned}
$$

(22) $\left\|\alpha^{i}(t)\right\|_{k(i)}^{*} \leq 1, \quad\left\|\beta^{i}(t)\right\|_{\ell(i)}^{*} \leq 1, \quad\left\|\gamma^{i}(t)\right\|_{m(i)}^{*} \leq 1$,

$$
\left\|\delta^{i}(t)\right\|_{n(i)}^{*} \leq 1, \quad t \in[a, b], \quad i \in \underline{r},
$$

$$
\left\|\zeta^{j}(t)\right\|_{p(j)}^{*} \leq 1, \quad\left\|\eta^{j}(t)\right\|_{q(j)}^{*} \leq 1, \quad t \in[a, b], \quad j \in \underline{s},
$$


(24)

$\lambda \in \Lambda, \mu \in \mathbb{R}_{+}, v \in P W S^{n}[a, b], w \in P W S_{+}^{s}[a, b], \alpha^{i} \in P W S^{k_{i}}[a, b]$,

$\beta^{i} \in P W S^{\ell_{i}}[a, b], \gamma^{i} \in P W S^{m_{i}}[a, b], \delta^{i} \in P W S^{n_{i}}[a, b]$,

$i \in \underline{r} ; \zeta^{j} \in P W S^{p_{j}}[a, b], \eta^{j} \in P W S^{q_{j}}[a, b], j \in \underline{s} ;$

$(\tilde{D} I) \quad$ Maximize $\mu$ subject to $(1),(4),(21)-(24)$, and

$$
\begin{array}{r}
\left\{\sum_{i=1}^{r} \lambda_{i}\left\{\nabla_{1} f_{i}(x, u, t)^{T}+\alpha^{i}(t)^{T} K_{i}(t)-\mu\left[\nabla_{1} g_{i}(x, u, t)^{T}-\gamma^{i}(t)^{T} M_{i}(t)\right]\right\}\right. \\
\left.+v(t)^{T} A(t)+\sum_{j=1}^{s} w_{j}(t)\left[\nabla_{1} h_{j}(x, u, t)^{T}+\zeta^{j}(t)^{T} P_{j}(t)\right]+D v(t)^{T}\right\}(\bar{x}-x) \geq 0
\end{array}
$$

for all $t \in[a, b]$ and $\bar{x} \in C^{n}[a, b]$ such that $(\bar{x}, u) \in \mathbb{F}$ for some $u \in$ $P W S^{m}[a, b]$,

$$
\begin{aligned}
& \left\{\sum_{i=1}^{r} \lambda_{i}\left\{\nabla_{2} f_{i}(x, u, t)^{T}+\beta^{i}(t)^{T} L_{i}(t)-\mu\left[\nabla_{2} g_{i}(x, u, t)^{T}-\delta^{i}(t)^{T} N_{i}(t)\right]\right\}\right. \\
& \left.+v(t)^{T} B(t)+\sum_{j=1}^{s} w_{j}(t)\left[\nabla_{2} h_{j}(x, u, t)^{T}+\eta^{j}(t)^{T} Q_{j}(t)\right]\right\}(\bar{u}-u) \geq 0
\end{aligned}
$$

for all $t \in[a, b]$ and $\bar{u} \in P W S^{m}[a, b]$ such that $(x, \bar{u}) \in \mathbb{F}$ for some $x \in C^{n}[a, b]$.

Comparing (DI) and $(\tilde{D} \mathrm{I})$, we observe that $(\tilde{D} \mathrm{I})$ is relatively more general than (DI) in the sense that any feasible solution of (DI) is also feasible for $(\tilde{D} \mathrm{I})$, but the converse is not necessarily true. Moreover, we see that (19) and (20) together form a system of $n+m$ equations, whereas (25) and (26) are two inequalities which in general cannot be expressed as equivalent systems of equations. Evidently, (DI) is preferable to $(\tilde{D} \mathrm{I})$ from a computational point of view because of the dependence of the latter on the feasible solution of $(\mathrm{P})$. However, despite these apparent differences, it turns out that all the duality results that can be established for (P)-(DI) are also valid for $(\mathrm{P})-(\tilde{D} \mathrm{I})$. Therefore, in the sequel we shall consider only the pair (P)-(DI).

For the sake of simplicity of notation, we shall henceforth let $\alpha=$ $\left(\alpha^{1}, \ldots, \alpha^{r}\right), \beta=\left(\beta^{1}, \ldots, \beta^{r}\right), \gamma=\left(\gamma^{1}, \ldots, \gamma^{r}\right), \delta=\left(\delta^{1}, \ldots, \delta^{r}\right), \zeta=$ $\left(\zeta^{1}, \ldots, \zeta^{s}\right)$, and $\eta=\left(\eta^{1}, \ldots, \eta^{s}\right)$.

The next two theorems show that (DI) is a dual problem for $(\mathrm{P})$. 
Theorem 4.1. (Weak Duality) Let $(\bar{x}, \bar{u})$ and $(x, u, \lambda, \mu, v, w, \alpha, \beta, \gamma$, $\delta, \zeta, \eta)$ be arbitrary feasible solutions of $(P)$ and $(D I)$, respectively. Then $\varphi(\bar{x}, \bar{u}) \geq \mu$.

Proof. Keeping in mind that $\lambda \geq 0, \mu \geq 0$, and $w(t) \geq 0$ for each $t \in[a, b]$, we have

$$
\begin{aligned}
& \sum_{i=1}^{r} \lambda_{i} \int_{a}^{b}\left\{f_{i}(\bar{x}, \bar{u}, t)+\left\|K_{i}(t) \bar{x}\right\|_{k(i)}+\left\|L_{i}(t) \bar{u}\right\|_{\ell(i)}-\mu\left[g_{i}(\bar{x}, \bar{u}, t)\right.\right. \\
& \left.\left.-\left\|M_{i}(t) \bar{x}\right\|_{m(i)}-\left\|N_{i}(t) \bar{u}\right\|_{n(i)}\right]\right\} d t-\sum_{i=1}^{r} \lambda_{i} \int_{a}^{b}\left\{f_{i}(x, u, t)\right. \\
& +\alpha^{i}(t)^{T} K_{i}(t) x+\beta^{i}(t)^{T} L_{i}(t) u-\mu\left[g_{i}(x, u, t)-\gamma^{i}(t)^{T} M_{i}(t) x\right. \\
& \left.\left.\quad-\delta^{i}(t)^{T} N_{i}(t) u\right]\right\} d t \\
& \geq \sum_{i=1}^{r} \lambda_{i} \int_{a}^{b}\left\{\nabla_{1} f_{i}(x, u, t)^{T}(\bar{x}-x)+\nabla_{2} f_{i}(x, u, t)^{T}(\bar{u}-u)\right. \\
& \quad-\mu\left[\nabla_{1} g_{i}(x, u, t)^{T}(\bar{x}-x)+\nabla_{2} g_{i}(x, u, t)^{T}(\bar{u}-u)\right]+\left\|K_{i}(t) \bar{x}\right\|_{k(i)} \\
& +\left\|L_{i}(t) \bar{u}\right\|_{\ell(i)}+\mu\left[\left\|M_{i}(t) \bar{x}\right\|_{m(i)}+\left\|N_{i}(t)^{i}\right\|_{n(i)}-\alpha^{i}(t)^{T} K_{i}(t) x\right. \\
& \left.\left.\quad-\beta^{i}(t)^{T} L_{i}(t) u-\mu \gamma^{i}(t)^{T} M_{i}(t) x-\mu \delta^{i}(t)^{T} N_{i}(t) u\right]\right\} d t
\end{aligned}
$$

(by the convexity of $f_{i}(., ., t)$ and $-g_{i}(., ., t), i \in \underline{r}$ )$$
=\int_{a}^{b}\left\{\sum _ { i = 1 } ^ { r } \lambda _ { i } \left\{\left[-\alpha^{i}(t)^{T} K_{i}(t)-\mu \gamma^{i}(t)^{T} M_{i}(t)\right](\bar{x}-x)\right.\right.
$$$$
+\left[-\beta^{i}(t)^{T} L_{i}(t) u-\mu \delta^{i}(t)^{T} N_{i}(t)\right](\bar{u}-u)+\left\|K_{i}(t) \bar{x}\right\|_{k(i)}+\left\|L_{i}(t) \bar{u}\right\|_{\ell(i)}
$$$$
+\mu\left[\left\|M_{i}(t) \bar{x}\right\|_{m(i)}+\left\|N_{i}(t) \bar{u}\right\|_{n(i)}-\alpha^{i}(t)^{T} K_{i}(t) x-\beta^{i}(t)^{T} L_{i}(t) u\right.
$$$$
\left.-\mu \gamma^{i}(t)^{T} M_{i}(t) x-\mu \delta^{i}(t)^{T} N_{i}(t) u\right]-\left\{v(t)^{T} A(t)\right.
$$$$
\left.+\sum_{j=1}^{s} w_{j}(t)\left[\nabla_{1} h_{j}(x, u, t)^{T}+\zeta^{j}(t)^{T} P_{j}(t)\right]+D v(t)^{T}\right\}(\bar{x}-x)
$$$$
\left.-\left\{v(t)^{T} B(t)+\sum_{j=1}^{s} w_{j}(t)\left[\nabla_{2} h_{j}(x, u, t)^{T}+\eta^{j}(t)^{T} Q_{j}(t)\right]\right\}\right\} d t
$$

(by (19) and (20))

$$
\geq \int_{a}^{b}\left\{\sum _ { i = 1 } ^ { r } \lambda _ { i } \left[-\left\|K_{i}(t) \bar{x}\right\|_{k(i)}\left\|\alpha^{i}(t)\right\|_{k(i)}^{*}-\mu\left\|M_{i}(t) \bar{x}\right\|_{m(i)}\left\|\gamma^{i}(t)\right\|_{m(i)}^{*}\right.\right.
$$


$-\left\|L_{i}(t) \bar{u}\right\|_{\ell(i)}\left\|\beta^{i}(t)\right\|_{\ell(i)}^{*}-\mu\left\|N_{i}(t) \bar{u}\right\|_{n(i)}\left\|\delta^{i}(t)\right\|_{n(i)}^{*}+\left\|K_{i}(t) \bar{x}\right\|_{k(i)}$

$\left.+\left\|L_{i}(t) \bar{u}\right\|_{\ell(i)}+\mu\left\|M_{i}(t) \bar{x}\right\|_{m(i)}+\mu\left\|N_{i}(t) \bar{u}\right\|_{n(i)}\right\}$

$+v(t)^{T}[D \bar{x}-D x-A(t)(\bar{x}-x)-B(t)(\bar{u}-u)]$

$-\sum_{j=1}^{s} w_{j}(t)\left[\left\|P_{j}(t) \bar{x}\right\|_{p(j)}\left\|\zeta^{j}(t)\right\|_{p(j)}^{*}+\left\|Q_{j}(t) \bar{u}\right\|_{q(j)}\left\|\eta^{j}(t)\right\|_{q(j)}^{*}\right]$

$+\sum_{j=1}^{s} w_{j}(t)\left[\zeta^{j}(t)^{T} P_{j}(t) x+\eta^{j}(t)^{T} Q_{j}(t) u\right]+\sum_{j=1}^{s} w_{j}(t)\left[h_{j}(x, u, t)\right.$

$\left.\left.-h_{j}(\bar{x}, \bar{u}, t)\right]\right\} d t$

(by Lemma 3.2, integration by parts, and convexity of $\left.h_{j}(., ., t), j \in \underline{s}\right)$

$\geq \int_{a}^{b}\left\{v(t)^{T}[D \bar{x}-A(t) \bar{x}-B(t) \bar{u}]-v(t)^{T}[D x-A(t) x-B(t) u]\right.$

$+\sum_{j=1}^{s} w_{j}(t)\left[h_{j}(x, u, t)+\zeta^{j}(t)^{T} P_{j}(t) x+\eta^{j}(t)^{T} Q_{j}(t) u\right]$

$\left.-\sum_{j=1}^{s} w_{j}(t)\left[h_{j}(\bar{x}, \bar{u}, t)+\left\|P_{j}(t) \bar{x}\right\|_{p(j)}+\left\|Q_{j}(t) \bar{u}\right\|_{q(j)}\right]\right\} d t$

(by (22) and (23))

$$
\begin{aligned}
\geq & \int_{a}^{b}\left\{-v(t)^{T}[D x-A(t) x-B(t) u]\right. \\
& \left.+\sum_{j=1}^{s} w_{j}(t)\left[h_{j}(x, u, t)+\zeta^{j}(t)^{T} P_{j}(t) x+\eta^{j}(t)^{T} Q_{j}(t) u\right]\right\} d t
\end{aligned}
$$

(by the primal feasibility of $(\vec{x}, \vec{u})$ ).

In view of (21) the above inequality reduces to

$$
\begin{aligned}
& \sum_{i=1}^{r} \lambda_{i} \int_{a}^{b}\left\{f_{i}(\bar{x}, \bar{u}, t)+\left\|K_{i}(t) \bar{x}\right\|_{k(i)}+\left\|L_{i}(t) \bar{u}\right\|_{\ell(i)}-\mu\left[g_{i}(\bar{x}, \bar{u}, t)\right.\right. \\
& \left.\left.-\left\|M_{i}(t) \bar{x}\right\|_{m(i)}-\left\|N_{i}(t) \bar{u}\right\|_{n(i)}\right]\right\} d t \geq 0 .
\end{aligned}
$$

Now using Lemma 3.3 and the above inequality, we see that 


$$
\begin{aligned}
\varphi(x, u) & =\max _{c \in \Lambda} \frac{\int_{a}^{b} \sum_{i=1}^{r} c_{i}\left[f_{i}(\bar{x}, \bar{u}, t)+\left\|K_{i}(t) \bar{x}\right\|_{k(i)}+\left\|L_{i}(t) \bar{u}\right\|_{\ell(i)}\right] d t}{\int_{a}^{b} \sum_{i=1}^{r} c_{i}\left[g_{i}(\bar{x}, \bar{u}, t)-\left\|M_{i}(t) \bar{x}\right\|_{m(i)}-\left\|N_{i}(t) \bar{u}\right\|_{n(i)}\right] d t} \\
& \geq \frac{\int_{a}^{b} \sum_{i=1}^{r} \lambda_{i}\left[f_{i}(\bar{x}, \bar{u}, t)+\left\|K_{i}(t) \bar{x}\right\|_{k(i)}+\left\|L_{i}(t) \bar{u}\right\|_{\ell(i)}\right] d t}{\int_{a}^{b} \sum_{i=1}^{r} \lambda_{i}\left[g_{i}(\bar{x}, \bar{u}, t)-\left\|M_{i}(t) \bar{x}\right\|_{m(i)}-\left\|N_{i}(t) \bar{u}\right\|_{n(i)}\right] d t} \\
& \geq \mu .
\end{aligned}
$$

Theorem 4.2. (Strong Duality) Let $\left(x^{*}, u^{*}\right)$ be an optimal solution of $(P)$. Then there exist $\lambda^{*} \in \Lambda, \mu^{*} \in \mathbb{R}_{+}, v^{*} \in P W S^{n}[a, b], w^{*} \in$ $P W S_{+}^{s}[a, b], \alpha^{* i} \in P W S^{k_{i}}[a, b], \beta^{* i} \in P W S^{\ell_{i}}[a, b], \gamma^{* i} \in P W S^{m_{i}}[a, b]$, $\delta^{* i} \in P W S^{n_{i}}[a, b], i \in \underline{r}, \zeta^{* j} \in P W S^{p_{j}}[a, b], \eta^{* j} \in P W S^{q_{j}}[a, b], j \in \underline{s}$, such that $z^{*} \equiv\left(x^{*}, u^{*}, \lambda^{*}, \mu^{*}, v^{*}, w^{*}, \alpha^{*}, \beta^{*}, \gamma^{*}, \delta^{*}, \zeta^{*}, \eta^{*}\right)$ is an optimal solution of $(D I)$ and $\varphi\left(x^{*}, u^{*}\right)=\mu^{*}$.

Proof. By Theorem 3.1, there exist $x^{*}, u^{*}, \lambda^{*}, \mu^{*}\left(=\varphi\left(x^{*}, u^{*}\right)\right), v^{*}, w^{*}$, $\alpha^{*}, \beta^{*}, \gamma^{*}, \delta^{*}, \zeta^{*}, \eta^{*}$, as specified above, such that $z^{*}$ is a feasible solution of (DI). Since $\varphi\left(x^{*}, u^{*}\right)=\mu^{*}$, optimality of $z^{*}$ for (DI) follows from Theorem 4.1.

We also have the following converse duality result for (P)-(DI).

THEOREm 4.3. (Strict Converse Duality) Let $\left(x^{*}, u^{*}\right)$ and $(\tilde{x}, \tilde{u}, \tilde{\lambda}, \tilde{\mu}$, $\tilde{v}, \tilde{w}, \tilde{\alpha}, \tilde{\beta}, \tilde{\gamma}, \tilde{\delta}, \tilde{\zeta}, \tilde{\eta})$ be optimal solutions of $(P)$ and $(D I)$, respectively, and assume that $f_{i}(., ., t)$ or $-g_{i}(., ., t)$ is strictly convex throughout $[a, b]$ for at least one index $i \in \underline{r}$ with the corresponding component $\tilde{\lambda}_{i}$ of $\tilde{\lambda}^{\prime}$ positive, or $h_{j}(., ., t)$ is strictly convex throughout $[a, b]$ for at least one $j \in \underline{s}$ with the corresponding component $\tilde{w}_{j}(t)$ of $\tilde{w}(t)$ positive on $[a, b]$. Then $(\tilde{x}(t), \tilde{u}(t))=\left(x^{*}(t), u^{*}(t)\right)$ for each $t \in[a, b]$, that is, $(\tilde{x}, \tilde{u})$ is an optimal solution of $(P)$, and $\varphi\left(x^{*}, u^{*}\right)=\tilde{\mu}$.

Proof. Suppose, on the contrary, that $(\tilde{x}(t), \tilde{u}(t)) \neq\left(x^{*}(t), u^{*}(t)\right)$ on a subset of $[a, b]$ with positive length. By Theorem 4.2, there exist $\lambda^{*}, \mu^{*}, v^{*}, w^{*}, \alpha^{*}, \beta^{*}, \gamma^{*}, \delta^{*}, \zeta^{*}, \eta^{*}$, as specified in Theorem 4.2, such that $\left(x^{*}, u^{*}, \lambda^{*}, \mu^{*}, v^{*}, w^{*}, \alpha^{*}, \beta^{*}, \gamma^{*}, \delta^{*}, \zeta^{*}, \eta^{*}\right)$ is an optimal solution of (DI) and $\varphi\left(x^{*}, u^{*}\right)=\mu^{*}$. Now proceeding as in the proof of Theorem 4.1 (with $(\bar{x}, \bar{u})$ replaced by $\left(x^{*}, u^{*}\right)$ and $(x, u, \lambda, v, w, \alpha, \beta, \gamma, \delta, \zeta, \eta)$ by $(\tilde{x}, \tilde{u}, \tilde{\lambda}, \tilde{\mu}, \tilde{v}, \tilde{w}, \tilde{\alpha}, \tilde{\beta}, \tilde{\gamma}, \tilde{\delta}, \tilde{\zeta}, \tilde{\eta})$, we arrive at the strict inequality

$$
\frac{\int_{a}^{b} \sum_{i=1}^{r} \tilde{\lambda}_{i}\left[f_{i}\left(x^{*}, u^{*}, t\right)+\left\|K_{i}(t) x^{*}\right\|_{k(i)}+\left\|L_{i}(t) u^{*}\right\|_{\ell(i)}\right] d t}{\int_{a}^{b} \sum_{i=1}^{r} \tilde{\lambda}_{i}\left[g_{i}\left(x^{*}, u^{*}, t\right)-\left\|M_{i}(t) x^{*}\right\|_{m(i)}-\left\|N_{i}(t) u^{*}\right\|_{n(i)}\right] d t}>\tilde{\mu} .
$$


Using this inequality and Lemma 3.3, we see, as in the proof of Theorem 4.1 , that $\varphi\left(x^{*}, u^{*}\right)>\tilde{\mu}$, which contradicts the fact that $\varphi\left(x^{*}, u^{*}\right)=\mu^{*}=$ $\tilde{\mu}$. Therefore, we must have $(\tilde{x}(t), \tilde{u}(t))=\left(x^{*}(t), u^{*}(t)\right)$ for all $t \in[a, b]$, and $\varphi\left(x^{*}, u^{*}\right)=\tilde{\mu}$.

\section{Duality model II}

In this section and the next, we formulate and discuss a number of parameter-free duality models for $(\mathrm{P})$. The forms and features of these duality models are based on Theorems 3.4 and 3.5. We begin with the following problem :

$(D I I) \quad$ Maximize $\frac{\int_{a}^{b} \sum_{i=1}^{r} \lambda_{i}\left[f_{i}(x, u, t)+\left\|K_{i}(t) x\right\|_{k(i)}+\left\|L_{i}(t) u\right\|_{\ell(i)}\right] d t}{\int_{a}^{b} \sum_{i=1}^{r} \lambda_{i}\left[g_{i}(x, u, t)-\left\|M_{i}(t) x\right\|_{m(i)}-\left\|N_{i}(t) u\right\|_{n(i)}\right] d t}$

subject to

$$
\begin{gathered}
x(a)=0, \quad x(b)=0 \\
\Psi(x, u, \lambda) \sum_{i=1}^{r} \lambda_{i}\left[\nabla_{1} f_{i}(x, u, t)+K_{i}(t)^{T} \alpha^{i}(t)\right] \\
-\Phi(x, u, \lambda) \sum_{i=1}^{r} \lambda_{i}\left[\nabla_{1} g_{i}(x, u, t)-M_{i}(t)^{T} \gamma^{i}(t)\right]+A(t)^{T} v(t) \\
+\sum_{j=1}^{s} w_{j}(t)\left[\nabla_{1} h_{j}(x, u, t)+P_{j}(t)^{T} \zeta^{j}(t)\right]+D v(t)=0, \quad t \in[a, b] \\
\Psi(x, u, \lambda) \sum_{i=1}^{r} \lambda_{i}\left[\nabla_{2} f_{i}(x, u, t)+L_{i}(t)^{T} \beta^{i}(t)\right] \\
-\Phi(x, u, \lambda) \sum_{i=1}^{r} \lambda_{i}\left[\nabla_{2} g_{i}(x, u, t)-N_{i}(t)^{T} \delta^{i}(t)\right]+B(t)^{T} v(t) \\
+\sum_{j=1}^{s} w_{j}(t)\left[\nabla_{2} h_{j}(x, u, t)+Q_{j}(t)^{T} \eta^{j}(t)\right]=0 \\
v(t)^{T}[-D x-A(t) x+B(t) u]+\sum_{j=1}^{s} w_{j}(t)\left[h_{j}(x, u, t)\right. \\
\left.+\left\|P_{j}(t) x\right\|_{p(j)}+\left\|Q_{j}(t) u\right\|_{q(j)}\right] \geq 0, \quad t \in[a, b]
\end{gathered}
$$


(31) $\left\|\alpha^{i}(t)\right\|_{k(i)}^{*} \leq 1,\left\|\beta^{i}(t)\right\|_{\ell(i)}^{*} \leq 1$,

$$
\left\|\gamma^{i}(t)\right\|_{m(i)}^{*} \leq 1, \quad\left\|\delta^{i}(t)\right\|_{n(i)}^{*} \leq 1, \quad t \in[a, b], \quad i \in \underline{r},
$$

$$
\left\|\zeta^{j}(t)\right\|_{p(j)}^{*} \leq 1, \quad\left\|\eta^{j}(t)\right\|_{q(j)}^{*} \leq 1, \quad t \in[a, b], \quad j \in \underline{s},
$$

$$
\begin{array}{r}
\alpha^{i}(t)^{T} K_{i}(t) x=\left\|K_{i}(t) x\right\|_{k(i)}, \quad \beta^{i}(t)^{T} L_{i}(t) u=\left\|L_{i}(t) u\right\|_{\ell(i)}, \\
\gamma^{i}(t)^{T} M_{i}(t) x=\left\|M_{i}(t) x\right\|_{m(i)}, \quad \delta^{i}(t)^{T} N_{i}(t) u=\left\|N_{i}(t) u\right\|_{n(i)}, \\
t \in[a, b], \quad i \in \underline{r},
\end{array}
$$

$$
\begin{aligned}
& \zeta^{j}(t)^{T} P_{j}(t) x=\left\|P_{j}(t) x\right\|_{p(j)}, \quad \eta^{j}(t)^{T} Q_{j}(t) u=\left\|Q_{j}(t) u\right\|_{q(j)}, \quad t \in[a, b], \\
& j \in \underline{s} \text {. }
\end{aligned}
$$

(35) $\lambda \in \Lambda, v \in P W S^{n}[a, b], w \in P W S_{+}^{s}[a, b], \alpha^{i} \in P W S^{k_{i}}[a, b]$,

$$
\begin{aligned}
\beta^{i} \in P W S^{\ell_{i}}[a, b], \gamma^{i} & \in P W S^{m_{i}}[a, b], \delta^{i} \in P W S^{n_{i}}[a, b], \\
i \in \underline{r} ; \zeta^{j} & \in P W S^{p_{j}}[a, b], \eta^{j} \in P W S^{q_{j}}[a, b], j \in \underline{s},
\end{aligned}
$$

where $\Phi$ and $\Psi$ are defined in Theorem 3.4;

$$
(\tilde{D} I I) \quad \text { Maximize } \frac{\int_{a}^{b} \sum_{i=1}^{r} \lambda_{i}\left[f_{i}(x, u, t)+\left\|K_{i}(t) x\right\|_{k(i)}+\left\|L_{i}(t) u\right\|_{\ell(i)}\right] d t}{\int_{a}^{b} \sum_{i=1}^{r} \lambda_{i}\left[g_{i}(x, u, t)-\left\|M_{i}(t) x\right\|_{m(i)}-\left\|N_{i}(t) u\right\|_{n(i)}\right] d t}
$$

subject to (27), (30)-(35), and

$$
\begin{aligned}
& \left\{\Psi(x, u, \lambda) \sum_{i=1}^{r} \lambda_{i}\left[\nabla_{1} f_{i}(x, u, t)^{T}+\alpha^{i}(t)^{T} K_{i}(t)\right]\right. \\
& -\Phi(x, u, \lambda) \sum_{i=1}^{r} \lambda_{i}\left[\nabla_{1} g_{i}(x, u, t)^{T}-\gamma^{i}(t)^{T} M_{i}(t)\right]+v(t)^{T} A(t) \\
& \left.+\sum_{j=1}^{s} w_{j}(t)\left[\nabla_{1} h_{j}(x, u, t)^{T}+\zeta^{j}(t)^{T} P_{j}(t)\right]+D v(t)^{T}\right\}(\bar{x}-x) \geq 0
\end{aligned}
$$


for all $t \in[a, b]$ and $\bar{x} \in C^{n}[a, b]$ such that $(\bar{x}, u) \in \mathbb{F}$ for some $u \in$ $P W S^{m}[a, b]$,

$$
\begin{aligned}
& \left\{\Psi ( x , u , \lambda ) \sum _ { i = 1 } ^ { r } \lambda _ { i } \left[\nabla_{2} f_{i}(x, u, t)^{T}+\beta^{i}(t)^{T} L_{i}(t)\right.\right. \\
& -\Phi(x, u, \lambda) \sum_{i=1}^{r} \lambda_{i}\left[\nabla_{2} g_{i}(x, u, t)^{T}-\delta^{i}(t)^{T} N_{i}(t)\right]+v(t)^{T} B(t) \\
& \left.+\sum_{j=1}^{s} w_{j}(t)\left[\nabla_{2} h_{j}(x, u, t)^{T}+\eta^{j}(t)^{T} Q_{j}(t)\right]\right\}(\bar{u}-u) \geq 0
\end{aligned}
$$

for all $t \in[a, b]$ and $\bar{u} \in P W S^{m}[a, b]$ such that $(x, \bar{u}) \in \mathbb{F}$ for some $x \in C^{n}[a, b]$.

The remarks made earlier about the relationships between (DI) and $(\tilde{D} \mathrm{I})$ are, of course, also applicable to (DII) and ( $\tilde{D} \mathrm{II})$.

Throughout this section and the next, it will be assumed that $\Phi(x, u$, $\lambda) \geq 0$ and $\Psi(x, u, \lambda)>0$ for all $x, u$, and $\lambda$ such that $(x, u, \lambda, v, w, \alpha, \beta$, $\gamma, \delta, \zeta, \eta)$ is a feasible solution of the dual problem under consideration.

The next two theorems show that (DII) is a dual problem for (P).

Theorem 5.1. (Weak Duality) Let $(\bar{x}, \bar{u})$ and $z \equiv(x, u, \lambda, v, w, \alpha, \beta$, $\gamma, \delta, \zeta, \eta)$ be arbitrary feasible solutions of $(P)$ and $(D I I)$, respectively. Then $\varphi(\bar{x}, \bar{u}) \geq \psi(z)$, where $\psi$ is the objective function of $(D I I)$.

Proof. Keeping in mind that $\lambda \geq 0, \Phi(x, u, \lambda) \geq 0, \Psi(x, u, \lambda)>0$, and $w(t) \geq 0$ for all $t \in[a, b]$, we have

$$
\begin{aligned}
& \int_{a}^{b} \sum_{i=1}^{r} \lambda_{i}\left[g_{i}(x, u, t)-\left\|M_{i}(t) x\right\|_{m(i)}\right. \\
& \left.-\left\|N_{i}(t) u\right\|_{n(i)}\right] d t \int_{a}^{b} \sum_{i=1}^{r} \lambda_{i}\left[f_{i}(\bar{x}, \bar{u}, t)+\left\|K_{i}(t) \bar{x}\right\|_{k(i)}\right. \\
& \left.+\left\|L_{i}(t) \bar{u}\right\|_{\ell(i)}\right] d t-\int_{a}^{b} \sum_{i=1}^{r} \lambda_{i}\left[f_{i}(x, u, t)+\left\|K_{i}(t) x\right\|_{k(i)}\right. \\
& \left.+\left\|L_{i}(t) u\right\|_{\ell(i)}\right] d t \int_{a}^{b} \sum_{i=1}^{r} \lambda_{i}\left[g_{i}(\bar{x}, \bar{u}, t)-\left\|M_{i}(t) \bar{x}\right\|_{m(i)}\right. \\
& \left.-\left\|N_{i}(t) \bar{u}\right\|_{n(i)}\right] d t \\
& =\Psi(x, u, \lambda)\left\{\int_{a}^{b} \sum_{i=1}^{r} \lambda_{i}\left[f_{i}(\bar{x}, \bar{u}, t)+\left\|K_{i}(t) \bar{x}\right\|_{k(i)}+\left\|L_{i}(t) \bar{u}\right\|_{\ell(i)}\right] d t\right.
\end{aligned}
$$




$$
\begin{aligned}
& \left.-\int_{a}^{b} \sum_{i=1}^{r} \lambda_{i}\left[f_{i}(x, u, t)+\left\|K_{i}(t) x\right\|_{k(i)}+\left\|L_{i}(t) u\right\|_{\ell(i)}\right] d t\right\} \\
& -\Phi(x, u, \lambda)\left\{\int_{a}^{b} \sum_{i=1}^{r} \lambda_{i}\left[g_{i}(\bar{x}, \bar{u}, t)-\left\|M_{i}(t) \bar{x}\right\|_{m(i)}-\left\|N_{i}(t) \bar{u}\right\|_{n(i)}\right] d t\right. \\
& \left.-\int_{a}^{b} \sum_{i=1}^{r} \lambda_{i}\left[g_{i}(x, u, t)-\left\|M_{i}(t) x\right\|_{m(i)}-\left\|N_{i}(t) u\right\|_{n(i)}\right] d t\right\} \\
& \geq \Psi(x, u, \lambda) \int_{a}^{b} \sum_{i=1}^{r} \lambda_{i}\left[\nabla_{1} f_{i}(x, u, t)^{T}(\bar{x}-x)+\nabla_{2} f_{i}(x, u, t)^{T}(\bar{u}-u)\right. \\
& \left.+\left\|K_{i}(t) \bar{x}\right\|_{k(i)}+\left\|L_{i}(t) \bar{u}\right\|_{\ell(i)}-\left\|K_{i}(t) x\right\|_{k(i)}-\left\|L_{i}(t) u\right\|_{\ell(i)}\right] d t \\
& -\Phi(x, u, \lambda) \int_{a}^{b} \sum_{i=1}^{r} \lambda_{i}\left[\nabla_{1} g_{i}(x, u, t)^{T}(\bar{x}-x)\right. \\
& +\nabla_{2} g_{i}(x, u, t)^{T}(\bar{u}-u)-\left\|M_{i}(t) \bar{x}\right\|_{m(i)}-\left\|N_{i}(t) \bar{u}\right\|_{n(i)} \\
& \left.+\left\|M_{i}(t) x\right\|_{m(i)}+\left\|N_{i}(t) u\right\|_{n(i)}\right] d t
\end{aligned}
$$

(by the convexity of $f_{i}(., ., t)$ and $-g_{i}(., ., t), i \in \underline{r}$ )

$$
\begin{aligned}
= & \int_{a}^{b}\left\{\Psi ( x , u , \lambda ) \sum _ { i = 1 } ^ { r } \lambda _ { i } \left[-\beta^{i}(t)^{T} L_{i}(t)(\bar{u}-u)-\alpha^{i}(t)^{T} K_{i}(t)(\bar{x}-x)\right.\right. \\
& \left.+\left\|K_{i}(t) \bar{x}\right\|_{k(i)}+\left\|L_{i}(t) \bar{u}\right\|_{\ell(i)}-\left\|K_{i}(t) x\right\|_{k(i)}-\left\|L_{i}(t) u\right\|_{\ell(i)}\right] \\
& -\left\{v(t)^{T} A(t)+\sum_{j=1}^{s} w_{j}(t)\left[\nabla_{1} h_{j}(x, u, t)^{T}+\zeta^{j}(t)^{T} P_{j}(t)\right]\right. \\
& \left.+D v(t)^{T}\right\}(\bar{x}-x)+\Phi(x, u, \lambda) \sum_{i=1}^{r} \lambda_{i}\left[-\delta^{i}(t)^{T} N_{i}(t)(\bar{u}-u)\right. \\
& \left.-\gamma^{i}(t)^{T} M_{i}(t)\right](\bar{x}-x)+\left\|M_{i}(t) \bar{x}\right\|_{m(i)}+\left\|N_{i}(t) \bar{u}\right\|_{n(i)}-\left\|M_{i}(t) x\right\|_{m(i)} \\
& \left.-\left\|N_{i}(t) u\right\|_{n(i)}\right]-\left\{v(t)^{T} B(t)+\sum_{j=1}^{s} w_{j}(t)\left[\nabla_{2} h_{j}(x, u, t)^{T}\right.\right. \\
& \left.\left.\left.+\eta^{j}(t)^{T} Q_{j}(t)\right]\right\}(\bar{u}-u)\right\} d t(b y(28) \text { and }(29)\} \\
\geq & \int_{a}^{b}\left\{\Psi ( x , u , \lambda ) \sum _ { i = 1 } ^ { r } \lambda _ { i } \left[-\left\|L_{i}(t) \bar{u}\right\|_{\ell(i)}\left\|\beta^{i}(t)\right\|_{\ell(i)}^{*}+\beta^{i}(t)^{T} L_{i}(t) u\right.\right. \\
& -\left\|K_{i}(t) \bar{x}\right\|_{k(i)}\left\|\alpha^{i}(t)\right\|_{k(i)}^{*}+\alpha^{i}(t)^{T} K_{i}(t) x+\left\|K_{i}(t) \bar{x}\right\|_{k(i)} \\
& \left.\left.+\left\|L_{i}(t) \bar{u}\right\|_{\ell(i)}\right]-\left\|K_{i}(t) x\right\|_{k(i)}-\left\|L_{i}(t) u\right\|_{\ell(i)}\right]-v(t)^{T} A(t)(\bar{x}-x)
\end{aligned}
$$




$$
\begin{aligned}
& -\sum_{j=1}^{s} w_{j}(t)\left[\nabla_{1} h_{j}(x, u, t)^{T}(\bar{x}-x)+\left\|P_{j}(t) \bar{x}\right\|_{p(j)}\left\|\zeta^{j}(t)\right\|_{p(j)}^{*}\right. \\
& -\zeta^{j}(t)^{T} P_{j}(t) x+v(t)^{T} D(\bar{x}-x) \\
& +\Phi(x, u, \lambda) \sum_{i=1}^{r} \lambda_{i}\left[-\left\|N_{i}(t) \bar{u}\right\|_{n(i)}\left\|\delta^{i}(t)\right\|_{n(i)}^{*}+\delta^{i}(t)^{T} N_{i}(t) u\right. \\
& -\left\|M_{i}(t) \bar{x}\right\|_{m(i)}\left\|\gamma^{i}(t)\right\|_{m(i)}^{*}+\gamma^{i}(t)^{T} M_{i}(t) x+\left\|M_{i}(t) \bar{x}\right\|_{m(i)} \\
& \left.+\left\|N_{i}(t) \bar{u}\right\|_{n(i)}-\left\|M_{i}(t) x\right\|_{m(i)}-\left\|N_{i}(t) u\right\|_{n(i)}\right]-v(t)^{T} B(t)(\bar{u}-u) \\
& -\sum_{j=1}^{s} w_{j}(t)\left[\nabla_{2} h_{j}(x, u, t)^{T}(\bar{u}-u)+\left\|Q_{j}(t) \bar{u}\right\|_{q(j)}\left\|\eta^{j}(t)\right\|_{q(j)}^{*}\right. \\
& \left.\left.-\eta^{j}(t)^{T} Q_{j}(t) u\right]\right\} d t
\end{aligned}
$$

(by Lemma 3.2 and integration by parts)

$$
\begin{aligned}
\geq & \int_{a}^{b}\left\{v(t)^{T}[D \bar{x}-A(t) \bar{x}-B(t) \bar{u}]-v(t)^{T}[D x-A(t) x-B(t) u]\right. \\
& -\sum_{j=1}^{s} \lambda_{i}\left[\nabla_{1} h_{j}(x, u, t)^{T}(\bar{x}-x)+\nabla_{2} h_{j}(x, u, t)^{T}(\bar{u}-u)+\left\|P_{j}(t) \bar{x}\right\|_{p(j)}\right. \\
& \left.\left.+\left\|Q_{j}(t) \bar{u}\right\|_{q(j)}-\zeta^{j}(t)^{T} P_{j}(t) x-\eta^{j}(t)^{T} Q_{j}(t) u\right]\right\} d t \text { (by (31)-(34)) } \\
\geq & \int_{a}^{b}\left\{v(t)^{T}[-D x+A(t) x+B(t) u]+\sum_{j=1}^{s} w_{j}(t)\left[h_{j}(x, u, t)\right.\right. \\
& \left.+\zeta^{j}(t)^{T} P_{j}(t) x+\eta^{j}(t)^{T} Q_{j}(t) u\right] \\
& \left.-\sum_{j=1}^{s} w_{j}(t)\left[h_{j}(\bar{x}, \bar{u}, t)+\left\|P_{j}(t) \bar{x}\right\|_{p(j)}+\left\|Q_{j}(t) \bar{u}\right\|_{q(j)}\right]\right\} d t
\end{aligned}
$$

(by the primal feasibility of $(\bar{x}, \bar{u})$ and convexity of $h_{j}(., ., t), j \in \underline{s}$ ) $\geq 0$ (by the primal feasibility of $(\bar{x}, \bar{u}),(30)$, and (33)).

Now using Lemma 3.3 and the above inequality, we see that

$$
\varphi(\bar{x}, \bar{u})=\max _{\mu \in \Lambda} \frac{\Phi(\bar{x}, \bar{u}, \mu)}{\Psi(\bar{x}, \bar{u}, \mu)} \geq \frac{\Phi(\bar{x}, \bar{u}, \lambda)}{\Psi(\bar{x}, \bar{u}, \lambda)} \geq \frac{\Phi(x, u, \lambda)}{\Psi(x, u, \lambda)}=\psi(z) .
$$

THEOREM 5.2. (Strong Duality) Let $\left(x^{*}, u^{*}\right)$ be an optimal solution of (P). Then there exist $\lambda^{*} \in \Lambda, v^{*} \in P W S^{n}[a, b], w^{*} \in P W S_{+}^{s}[a, b], \alpha^{* i} \in$ 
$P W S^{k_{i}}[a, b], \beta^{* i} \in P W S^{\ell_{i}}[a, b], \gamma^{* i} \in P W S^{m_{i}}[a, b], \delta^{* i} \in P W S^{n_{i}}[a, b]$, $i \in \underline{r}, \zeta^{* j} \in P W S^{p_{j}}[a, b], \eta^{* j} \in P W S^{q_{j}}[a, b], j \in \underline{s}$, such that $z^{*} \equiv$ $\left(x^{*}, u^{*}, \lambda^{*}, v^{*}, w^{*}, \alpha^{*}, \beta^{*}, \gamma^{*}, \delta^{*}, \zeta^{*}, \eta^{*}\right)$ is an optimal solution of $(D I I)$ and $\varphi\left(x^{*}, u^{*}\right)=\psi\left(z^{*}\right)$.

Proof. By Theorem 3.4, there exist $\lambda^{*}, v^{*}, w^{*}, \alpha^{*}, \beta^{*}, \gamma^{*}, \delta^{*}, \zeta^{*}, \eta^{*}$, as specified above, such that $z^{*}$ is a feasible solution of (DII). Since $\varphi\left(x^{*}, u^{*}\right)$ $=\Phi\left(x^{*}, u^{*}, \lambda^{*}\right) / \Psi\left(x^{*}, u^{*}, \lambda^{*}\right)=\psi\left(z^{*}\right)$, optimality of $z^{*}$ for (DII) follows from Theorem 5.1.

ThEOREM 5.3. (Strict Converse Duality) Let $\left(x^{*}, u^{*}\right)$ and $\tilde{z} \equiv(\tilde{x}, \tilde{u}$, $\tilde{\lambda}, \tilde{v}, \tilde{w}, \tilde{\alpha}, \tilde{\beta}, \tilde{\gamma}, \tilde{\delta}, \tilde{\zeta}, \tilde{\eta})$ be optimal solutions of $(P)$ and (DII), respectively, and assume that $f_{i}(., ., t)$ or $-g_{i}(., ., t)$ is strictly convex throughout $[a, b]$ for at least one index $i \in \underline{r}$ with the corresponding component $\tilde{\lambda}_{i}$ of $\tilde{\lambda}$ positive, or $h_{j}(., ., t)$ is strictly convex throughout $[a, b]$ for at least one $j \in \underline{s}$ with the corresponding component $\tilde{w}_{j}(t)$ of $\tilde{w}(t)$ positive on $[a, b]$. Then $(\tilde{x}(t), \tilde{u}(t))=\left(x^{*}(t), u^{*}(t)\right)$ for each $t \in[a, b]$, that is, $(\tilde{x}, \tilde{u})$ is an optimal solution of $(P)$, and $\varphi\left(x^{*}, u^{*}\right)=\psi(\tilde{z})$.

Proof. Suppose, on the contrary, that $(\tilde{x}(t), \tilde{u}(t)) \neq\left(x^{*}(t), u^{*}(t)\right)$ on a subset of $[a, b]$ with positive length. By Theorem 5.2, there exist $\lambda^{*}, v^{*}, w^{*}, \alpha^{*}, \beta^{*}, \gamma^{*}, \delta^{*}, \zeta^{*}, \eta^{*}$, as specified in Theorem 5.2, such that $z^{*} \equiv\left(x^{*}, u^{*}, \lambda^{*}, v^{*}, w^{*}, \alpha^{*}, \beta^{*}, \gamma^{*}, \delta^{*}, \zeta^{*}, \eta^{*}\right)$ is an optimal solution of (DII) and $\varphi\left(x^{*}, u^{*}\right)=\psi\left(z^{*}\right)$. Now proceeding as in the proof of Theorem 5.1 (with $(\bar{x}, \bar{u})$ replaced by $\left(x^{*}, u^{*}\right)$ and $z$ by $\tilde{z}$ ), we arrive at the strict inequality

$$
\frac{\Phi\left(x^{*}, u^{*}, \tilde{\lambda}\right)}{\Psi\left(x^{*}, u^{*}, \tilde{\lambda}\right)}>\frac{\Phi(\tilde{x}, \tilde{u}, \tilde{\lambda})}{\Psi(\tilde{x}, \tilde{u}, \tilde{\lambda})} .
$$

Using this inequality and Lemma 3.3 , we see, as in the proof of Theorem 3.2, that $\varphi\left(x^{*}, u^{*}\right)>\psi(\tilde{z})$, which contradicts the fact that $\varphi\left(x^{*}, u^{*}\right)=\psi\left(z^{*}\right)=\psi(\tilde{z})$. Therefore, we must have $(\tilde{x}(t), \tilde{u}(t))=$ $\left(x^{*}(t), u^{*}(t)\right)$ for all $t \in[a, b]$, and $\varphi\left(x^{*}, u^{*}\right)=\psi(\tilde{z})$.

The formulation of the dual problems (DII) and $(\tilde{D} I I)$ was based directly on the form of the optimality conditions of Theorem 3.4. However, a careful examination of the proofs of Theorems 5.1 and 5.2 will readily reveal the fact that the constraints (33) and (34) are essentially redundant in the sense that their deletion will not invalidate the duality results. More specifically, it can be shown that the following streamlined versions of (DII) and ( $\tilde{D} I I)$ obtained by dropping (33) and (34), and then modifying the objective functions and the remaining constraints of 
(DII) and ( $\tilde{D} I I)$ accordingly, are also bona fide dual problems for (P): (DIIA) Maximize $\frac{\int_{a}^{b} \sum_{i=1}^{r} \lambda_{i}\left[f_{i}(x, u, t)+\alpha^{i}(t)^{T} K_{i}(t) x+\beta^{i}(t)^{T} L_{i}(t) u\right] d t}{\int_{a}^{b} \sum_{i=1}^{r} \lambda_{i}\left[g_{i}(x, u, t)-\gamma^{i}(t)^{T} M_{i}(t) x-\delta^{i}(t)^{T} N_{i}(t) u\right] d t}$ subject to (27), (35), and

$$
\begin{aligned}
& \sum_{i=1}^{r} \lambda_{i}\left\{\Gamma(x, u, \lambda, \gamma, \delta)\left[\nabla_{1} f_{i}(x, u, t)+K_{i}(t)^{T} \alpha^{i}(t)\right]\right. \\
& \left.-\Pi(x, u, \lambda, \alpha, \beta)\left[\nabla_{1} g_{i}(x, u, t)-M_{i}(t)^{T} \gamma^{i}(t)\right]\right\}+A(t)^{T} v(t) \\
& +\sum_{j=1}^{s} w_{j}(t)\left[\nabla_{1} h_{j}(x, u, t)+P_{j}(t)^{T} \zeta^{j}(t)\right] \\
& +D v(t)=0, \quad t \in[a, b]
\end{aligned}
$$

$$
\begin{aligned}
& \sum_{i=1}^{r} \lambda_{i}\left\{\Gamma(x, u, \lambda, \gamma, \delta)\left[\nabla_{2} f_{i}(x, u, t)+L_{i}(t)^{T} \beta^{i}(t)\right]\right. \\
& \left.-\Pi(x, u, \lambda, \gamma, \delta)\left[\nabla_{2} g_{i}(x, u, t)-N_{i}(t)^{T} \delta^{i}(t)\right]\right\}+B(t)^{T} v(t) \\
& \quad+\sum_{j=1}^{s} w_{j}(t)\left[\nabla_{2} h_{j}(x, u, t)+Q_{j}(t)^{T} \eta^{j}(t)\right]=0, \quad t \in[a, b]
\end{aligned}
$$

(38) $v(t)^{T}[-D x-A(t) x-B(t) u]+\sum_{j=1}^{s} w_{j}(t)\left[h_{j}(x, u, t)\right.$

$$
\left.+\zeta^{j}(t)^{T} P_{j}(t) x+\eta^{j}(t)^{T} Q_{j}(t) u\right] \geq 0, t \in[a, b]
$$

(39) $\left\|\alpha^{i}(t)\right\|_{k(i)}^{*} \leq 1, \quad\left\|\beta^{i}(t)\right\|_{\ell(i)}^{*} \leq 1$,

$$
\left\|\gamma^{i}(t)\right\|_{m(i)}^{*} \leq 1, \quad\left\|\delta^{i}(t)\right\|_{n(i)}^{*} \leq 1, \quad t \in[a, b], \quad i \in \underline{r},
$$

$$
\left\|\zeta^{j}(t)\right\|_{p(j)}^{*} \leq 1, \quad\left\|\eta^{j}(t)\right\|_{q(j)}^{*} \leq 1, \quad t \in[a, b], \quad j \in \underline{s}
$$

where

$$
\Pi(x, u, \lambda, \alpha, \beta)=\int_{a}^{b} \sum_{i=1}^{r} \lambda_{i}\left[f_{i}(x, u, t)+\alpha^{i}(t)^{T} K_{i}(t) x+\beta^{i}(t)^{T} L_{i}(t) u\right] d t
$$

and

$$
\Gamma(x, u, \lambda, \gamma, \delta)=\int_{a}^{b} \sum_{i=1}^{r} \lambda_{i}\left[g_{i}(x, u, t)-\gamma^{i}(t)^{T} M_{i}(t) x-\delta^{i}(t)^{T} N_{i}(t) u\right] d t
$$


$(\tilde{D} I I A) \quad$ Maximize $\frac{\int_{a}^{b} \sum_{i=1}^{r} \lambda_{i}\left[f_{i}(x, u, t)+\alpha^{i}(t)^{T} K_{i}(t) x+\beta^{i}(t)^{T} L_{i}(t) u\right] d t}{\int_{a}^{b} \sum_{i=1}^{r} \lambda_{i}\left[g_{i}(x, u, t)-\gamma^{i}(t)^{T} M_{i}(t) x-\delta^{i}(t)^{T} N_{i}(t) u\right] d t}$

subject to $(27),(35),(38)-(40)$, and

$$
\begin{aligned}
& \left\{\sum _ { i = 1 } ^ { r } \lambda _ { i } \left\{\Gamma(x, u, \lambda, \gamma, \delta)\left[\nabla_{1} f_{i}(x, u, t)^{T}+\alpha^{i}(t)^{T} K_{i}(t)\right]\right.\right. \\
& \left.\quad-\Pi(x, u, \lambda, \alpha, \beta)\left[\nabla_{1} g_{i}(x, u, t)^{T}-\gamma^{i}(t)^{T} M_{i}(t)\right]\right\}+v(t)^{T} A(t) \\
& \left.\quad+\sum_{j=1}^{s} w_{j}(t)\left[\nabla_{1} h_{j}(x, u, t)^{T}+\zeta^{j}(t)^{T} P_{j}(t)\right]+D v(t)^{T}\right\}(\bar{x}-x) \geq 0
\end{aligned}
$$

for all $t \in[a, b]$ and $\bar{x} \in C^{n}[a, b]$ such that $(\bar{x}, u) \in \mathbb{F}$ for some $u \in$ $P W S^{m}[a, b]$,

$$
\begin{aligned}
& \left\{\sum _ { i = 1 } ^ { r } \lambda _ { i } \left\{\Gamma ( x , u , \lambda , \gamma , \delta ) \left[\nabla_{2} f_{i}(x, u, t)^{T}+\beta^{i}(t)^{T} L_{i}(t)\right.\right.\right. \\
& \left.-\Pi(x, u, \lambda, \alpha, \beta)\left[\nabla_{2} g_{i}(x, u, t)^{T}-\delta^{i}(t)^{T} N_{i}(t)\right]\right\}+v(t)^{T} B(t) \\
& \left.+\sum_{j=1}^{s} w_{j}(t)\left[\nabla_{2} h_{j}(x, u, t)^{T}+\eta^{j}(t)^{T} Q_{j}(t)\right]\right\}(\bar{u}-u) \geq 0
\end{aligned}
$$

for all $t \in[a, b]$ and $\bar{u} \in P W S^{m}[a, b]$ such that $(x, \bar{u}) \in \mathbb{F}$ for some $x \in C^{n}[a, b]$.

The remarks made earlier about the relationships between (DI) and

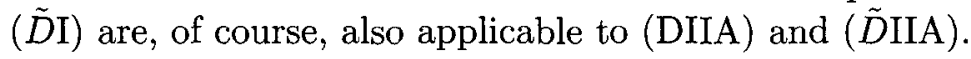

We next show that (DIIA) and (DIIA) are indeed dual problems for (P).

Throughout this section and the next, it will be assumed that $\Pi(x, u$, $\lambda, \alpha, \beta) \geq 0$ and $\Gamma(x, u, \lambda, \gamma, \delta)>0$ for all $x, u, \lambda, \alpha, \beta, \gamma$, and $\delta$, such that $(x, u, \lambda, v, w, \alpha, \beta, \gamma, \delta, \zeta, \eta)$ is a feasible solution of the dual problem under consideration.

Theorem 5.4. (Weak Duality) Let $(\bar{x}, \bar{u})$ and $z \equiv(x, u, \lambda, v, w, \alpha, \beta$, $\gamma, \delta, \zeta, \eta)$ be arbitrary feasible solutions of $(P)$ and $(D I I A)$, respectively. Then $\varphi(\bar{x}, \bar{u}) \geq \omega(z)$, where $\omega$ is the objective function of (DIIA). 
Proof. Keeping in mind that $\lambda \geq 0, \Pi(x, u, \lambda, \alpha, \beta) \geq 0, \Gamma(x, u, \lambda, \gamma$, $\delta)>0$, and $w(t) \geq 0$ for all $t \in[a, b]$, we have

$$
\begin{aligned}
& \Gamma(x, u, \lambda, \gamma, \delta) \Phi(\bar{x}, \bar{u}, \lambda)-\Pi(x, u, \lambda, \alpha, \beta) \Psi(\bar{x}, \bar{u}, \lambda) \\
= & \int_{a}^{b} \sum_{i=1}^{r} \lambda_{i}\left[g_{i}(x, u, t)-\gamma^{i}(t)^{T} M_{i}(t) x\right. \\
& \left.-\delta^{i}(t)^{T} N_{i}(t) u\right] d t \int_{a}^{b} \sum_{i=1}^{r} \lambda_{i}\left[f_{i}(\bar{x}, \bar{u}, t)+\left\|K_{i}(t) \bar{x}\right\|_{k(i)}\right. \\
& \left.+\left\|L_{i}(t) \bar{u}\right\|_{\ell(i)}\right] d t-\int_{a}^{b} \sum_{i=1}^{r} \lambda_{i}\left[f_{i}(x, u, t)+\alpha^{i}(t)^{T} K_{i}(t) x\right. \\
& \left.+\beta^{i}(t)^{T} L_{i}(t) u\right] d t \int_{a}^{b} \sum_{i=1}^{r} \lambda_{i}\left[g_{i}(\bar{x}, \bar{u}, t)\right. \\
& \left.-\left\|M_{i}(t) \bar{x}\right\|_{m(i)}-\left\|N_{i}(t) \bar{u}\right\|_{n(i)}\right] d t \\
= & \Gamma(x, u, \lambda, \gamma, \delta) \int_{a}^{b} \sum_{i=1}^{r} \lambda_{i}\left\{f_{i}(\bar{x}, \bar{u}, t)+\left\|K_{i}(t) \bar{x}\right\|_{k(i)}\right. \\
& \left.+\left\|L_{i}(t) \bar{u}\right\|_{\ell(i)}-\left[f_{i}(x, u, t)+\alpha^{i}(t)^{T} K_{i}(t) x+\beta^{i}(t)^{T} L_{i}(t) u\right] d t\right\} \\
& -\Pi(x, u, \lambda, \alpha, \beta) \int_{a}^{b} \sum_{i=1}^{r} \lambda_{i}\left\{g_{i}(\bar{x}, \bar{u}, t)\right. \\
& \left.-\left\|M_{i}(t) \bar{x}\right\|_{m(i)}-\left\|N_{i}(t) \bar{u}\right\|_{n(i)}\right] \\
& \left.-\left[g_{i}(x, u, t)-\gamma^{i}(t)^{T} M_{i}(t) x-\delta^{i}(t)^{T} N_{i}(t) u\right] d t\right\} \\
\geq & \int_{a}^{b} \sum_{i=1}^{r} \lambda_{i}\left\{\Gamma ( x , u , \lambda , \gamma , \delta ) \left[\nabla_{1} f_{i}(x, u, t)^{T}(\bar{x}-x)\right.\right. \\
& +\nabla_{2} f_{i}(x, u, t)^{T}(\bar{u}-u)+\left\|K_{i}(t) \bar{x}\right\|_{k(i)}+\left\|L_{i}(t) \bar{u}\right\|_{\ell(i)}-\alpha^{i}(t)^{T} K_{i}(t) x \\
& \left.-\beta^{i}(t)^{T} L_{i}(t) u\right]-\Pi(x, u, \lambda, \alpha, \beta)\left[\nabla_{1} g_{i}(x, u, t)^{T}(\bar{x}-x)\right. \\
& +\nabla_{2} g_{i}(x, u, t)^{T}(\bar{u}-u)-\left\|M_{i}(t) \bar{x}\right\|_{m(i)}-\left\|N_{i}(t) \bar{u}\right\|_{n(i)} \\
& \left.\left.+\gamma^{i}(t)^{T} M_{i}(t) x+\delta^{i}(t)^{T} N_{i}(t) u\right]\right\} d t \\
& (b y t h e(c o n)
\end{aligned}
$$

(by the convexity of $f_{i}(., ., t)$ and $-g_{i}(., ., t), i \in \underline{r}$ )$$
=\int_{a}^{b}\left\{\Gamma ( x , u , \lambda , \gamma , \delta ) \sum _ { i = 1 } ^ { r } \lambda _ { i } \left[-\alpha^{i}(t)^{T} K_{i}(t) \bar{x}-\beta^{i}(t)^{T} L_{i}(t) \bar{u}\right.\right.
$$ 


$$
\begin{aligned}
& \left.+\left\|K_{i}(t) \bar{x}\right\|_{k(i)}+\left\|L_{i}(t) \bar{u}\right\|_{\ell(i)}\right]-\left\{v(t)^{T} A(t)\right. \\
& \left.+\sum_{j=1}^{s} w_{j}(t)\left[\nabla_{1} h_{j}(x, u, t)^{T}+\zeta^{j}(t)^{T} P_{j}(t)\right]+D v(t)^{T}\right\}(\bar{x}-x) \\
& -\Pi(x, u, \lambda, \alpha, \beta) \sum_{i=1}^{r} \lambda_{i}\left[\gamma^{i}(t)^{T} M_{i}(t) \bar{x}+\delta^{i}(t)^{T} N_{i}(t) \bar{u}-\left\|M_{i}(t) \bar{x}\right\|_{m(i)}\right. \\
& \left.-\left\|N_{i}(t) \bar{u}\right\|_{n(i)}\right] \\
& \left.-\left\{v(t)^{T} B(t)+\sum_{j=1}^{s} w_{j}(t)\left[\nabla_{2} h_{j}(x, u, t)^{T}+\eta^{j}(t)^{T} Q_{j}(t)\right]\right\}(\bar{u}-u)\right\}
\end{aligned}
$$

(by (36) and (37))

$$
\begin{aligned}
\geq & \int_{a}^{b}\left\{\Gamma ( x , u , \lambda , \gamma , \delta ) \sum _ { i = 1 } ^ { r } \lambda _ { i } \left[-\left\|K_{i}(t) \bar{x}\right\|_{k(i)}\left\|\alpha^{i}(t)\right\|_{k(i)}^{*}\right.\right. \\
& \left.-\left\|L_{i}(t) \bar{u}\right\|_{\ell(i)}\left\|\beta^{i}(t)\right\|_{\ell(i)}^{*}+\left\|K_{i}(t) \bar{x}\right\|_{k(i)}+\left\|L_{i}(t) \bar{u}\right\|_{\ell(i)}\right] \\
& +\left[D v(t)^{T}+v(t)^{T} A(t)\right](\bar{x}-x)-v(t)^{T} B(t)(\bar{u}-u) \\
& -\sum_{j=1}^{s} w_{j}(t)\left[\nabla_{1} h_{j}(x, u, t)^{T}(\bar{x}-x)+\nabla_{2} h_{j}(x, u, t)^{T}(\bar{u}-u)\right. \\
& \left.+\left\|P_{j}(t) \bar{x}\right\|_{p(j)}\left\|\zeta^{j}(t)\right\|_{p(j)}^{*}-\zeta_{j}(t)^{T} P_{j}(t) x+\left\|Q_{j}(t) \bar{u}\right\|_{q(j)} \| \eta^{j} t\right) \|_{q(j)}^{*} \\
& \left.-\eta^{j}(t)^{T} Q_{j}(t) u\right]-\Pi(x, u, \lambda, \alpha, \beta) \sum_{i=1}^{r} \lambda_{i}\left[\left\|M_{i}(t) \bar{x}\right\|_{m(i)}\left\|\gamma^{i}(t)\right\|_{m(i)}^{*}\right. \\
& \left.\left.+\left\|N_{i}(t) \bar{u}\right\|_{n(i)}\left\|\delta^{i}(t)\right\|_{n(i)}^{*}-\left\|M_{i}(t) \bar{x}\right\|_{m(i)}-\left\|N_{i}(t) \bar{u}\right\|_{n(i)}\right]\right\} d t
\end{aligned}
$$

(by Lemma 3.2)

$$
\begin{aligned}
\geq & \int_{a}^{b}\left\{v(t)^{T}[D \bar{x}-A(t) \bar{x}-B(t) \bar{u}]-v(t)^{T}[-D x+A(t) x+B(t) u]\right. \\
& -\sum_{j=1}^{s} w_{j}(t)\left[\nabla_{1} h_{j}(x, u, t)^{T}(\bar{x}-x)+\nabla_{2} h_{j}(x, u, t)^{T}(\bar{u}-u)\right. \\
& \left.\left.\left.+\left\|P_{j}(t) \bar{x}\right\|_{p(j)}+\left\|Q_{j}(t) \bar{u}\right\|_{q(j)}\right]-\zeta_{j}(t)^{T} P_{j}(t) x-\eta^{j}(t)^{T} Q_{j}(t) u\right]\right\} d t
\end{aligned}
$$

(by (39), (40), and integration by parts)

$$
\geq \int_{a}^{b}\left\{v(t)^{T}[-D x+A(t) x+B(t) u]\right.
$$




$$
\begin{aligned}
& +\sum_{j=1}^{s} w_{j}(t)\left[h_{j}(x, u, t)+\zeta_{j}(t)^{T} P_{j}(t) x+\eta^{j}(t)^{T} Q_{j}(t) u\right] \\
& \left.-\sum_{j=1}^{s} w_{j}(t)\left[h_{j}(\bar{x}, \bar{u}, t)+\left\|P_{j}(t) \bar{x}\right\|_{p(j)}+\left\|Q_{j}(t) \bar{u}\right\|_{q(j)}\right]\right\} d t
\end{aligned}
$$

(by the primal feasibility of $(\vec{x}, \bar{u})$ and convexity of $h_{j}(., ., t), j \in \underline{s}$ ) $\geq 0$ (by the primal feasibility of $(\bar{x}, \bar{u})$ and $(38)$ ).

Now from Lemma 3.3 and the above inequality we see that

$$
\varphi(\bar{x}, \bar{u})=\max _{\mu \in \Lambda} \frac{\Phi(\bar{x}, \bar{u}, \mu)}{\Psi(\bar{x}, \bar{u}, \mu)} \geq \frac{\Phi(\bar{x}, \bar{u}, \lambda)}{\Psi(\bar{x}, \bar{u}, \lambda)} \geq \frac{\Pi(x, u, \lambda, \alpha, \beta)}{\Gamma(x, u, \lambda, \gamma, \delta)}=\omega(z) .
$$

THEOREM 5.5. (Strong Duality) Let $\left(x^{*}, u^{*}\right)$ be an optimal solution of (P). Then there exist $\lambda^{*} \in \Lambda, v^{*} \in P W S^{n}[a, b], w^{*} \in P W S_{+}^{s}[a, b], \alpha^{* i} \in$ $P W S^{k_{i}}[a, b], \beta^{* i} \in P W S^{\ell_{i}}[a, b], \gamma^{* i} \in P W S^{m_{i}}[a, b], \delta^{* i} \in P W S^{n_{i}}[a, b]$, $i \in \underline{r}, \zeta^{* j} \in P W S^{p_{j}}[a, b], \eta^{* j} \in P W S^{q_{j}}[a, b], j \in \underline{s}$, such that

$$
z^{*} \equiv\left(x^{*}, u^{*}, \lambda^{*}, v^{*}, w^{*}, \alpha^{*}, \beta^{*}, \gamma^{*}, \delta^{*}, \zeta^{*}, \eta^{*}\right)
$$

is an optimal solution of (DIIA) and $\varphi\left(x^{*}, u^{*}\right)=\omega\left(z^{*}\right)$.

Proof. By Theorem 3.4, there exist $\lambda^{*}, v^{*}, w^{*}, \alpha^{*}, \beta^{*}, \gamma^{*}, \delta^{*}, \zeta^{*}, \eta^{*}$, as specified above, such that $z^{*}$ is a feasible solution of (DIIA) and $\varphi\left(x^{*}, u^{*}\right)$ $=\Phi\left(x^{*}, u^{*}, \lambda^{*}\right) / \Psi\left(x^{*}, u^{*}, \lambda^{*}\right)=\omega\left(z^{*}\right)$, because

$$
\begin{aligned}
& \alpha^{* i}(t)^{T} K_{i}(t) x^{*}=\left\|K_{i}(t) x^{*}\right\|_{k(i)}, \beta^{* i}(t)^{T} L_{i}(t) u^{*} \\
= & \left\|L_{i}(t) u^{*}\right\|_{\ell(i)}, \gamma^{* i}(t)^{T} M_{i}(t) x^{*}=\left\|M_{i}(t) x^{*}\right\|_{m(i)},
\end{aligned}
$$

and $\delta^{* i}(t)^{T} N_{i}(t) u^{*}=\left\|N_{i}(t) u^{*}\right\|_{n(i)}$ for all $t \in[a, b]$ and all $i \in \underline{r}$. Now optimality of $z^{*}$ for (DIIA) follows from Theorem 5.4.

Theorem 5.6. (Strict Converse Duality) Let $\left(x^{*}, u^{*}\right)$ and $\tilde{z} \equiv(\tilde{x}, \tilde{u}$, $\tilde{\lambda}, \tilde{v}, \tilde{w}, \tilde{\alpha}, \tilde{\beta}, \tilde{\gamma}, \tilde{\delta}, \tilde{\zeta}, \tilde{\eta})$, be optimal solutions of $(P)$ and (DIIA), respectively, and assume that $f_{i}(., ., t)$ or $-g_{i}(., ., t)$ is strictly convex throughout $[a, b]$ for at least one index $i \in \underline{r}$ with the corresponding component $\tilde{\lambda}_{i}$ of $\tilde{\lambda}$ positive, or $h_{j}(., ., t)$ is strictly convex throughout $[a, b]$ for at least one $j \in \underline{s}$ with the corresponding component $\tilde{w}_{j}(t)$ of $\tilde{w}(t)$ positive on $[a, b]$. Then $(\tilde{x}(t), \tilde{u}(t))=\left(x^{*}(t), u^{*}(t)\right)$ for each $t \in[a, b]$, that is, $(\tilde{x}, \tilde{u})$ is an optimal solution of $(P)$, and $\varphi\left(x^{*}, u^{*}\right)=\omega(\tilde{z})$.

Proof. Suppose, on the contrary, that $(\tilde{x}(t), \tilde{u}(t)) \neq\left(x^{*}(t), u^{*}(t)\right)$ on a subset of $[a, b]$ with positive length. By Theorem 5.5, there exist 
$\lambda^{*}, v^{*}, w^{*}, \alpha^{*}, \beta^{*}, \gamma^{*}, \delta^{*}, \zeta^{*}, \eta^{*}$, as specified in Theorem 5.5, such that $z^{*} \equiv\left(x^{*}, u^{*}, \lambda^{*}, v^{*}, w^{*}, \alpha^{*}, \beta^{*}, \gamma^{*}, \delta^{*}, \zeta^{*}, \eta^{*}\right)$ is an optimal solution of (DIIA) and $\varphi\left(x^{*}, u^{*}\right)=\omega\left(z^{*}\right)$. Now proceeding as in the proof of Theorem 5.4 (with $(\bar{x}, \bar{u})$ replaced by $\left(x^{*}, u^{*}\right)$ and $z$ by $\tilde{z}$ ), we arrive at the strict inequality

$$
\frac{\Phi\left(x^{*}, u^{*}, \tilde{\lambda}\right)}{\Psi\left(x^{*}, u^{*}, \tilde{\lambda}\right)}>\frac{\Phi(\tilde{x}, \tilde{u}, \tilde{\lambda})}{\Psi(\tilde{x}, \tilde{u}, \tilde{\lambda})} .
$$

Using this inequality and Lemma 3.3 , we see, as in the proof of Theorem 5.4, that $\varphi\left(x^{*}, u^{*}\right)>\omega(\tilde{z})$, which contradicts the fact that $\varphi\left(x^{*}, u^{*}\right)=\omega\left(z^{*}\right)=\omega(\tilde{z})$. Therefore, we must have $(\tilde{x}(t), \tilde{u}(t))=$ $\left(x^{*}(t), u^{*}(t)\right)$ for all $t \in[a, b]$, and $\varphi\left(x^{*}, u^{*}\right)=\omega(\tilde{z})$.

\section{Duality model III}

We shall continue our discussion of duality for $(\mathrm{P})$ in this section by considering the following variants of (DII) and ( $\tilde{D} \mathrm{II})$ :

$$
(D I I I) \quad \text { Maximize } \frac{\Phi(x, u, \lambda)+\Omega(x, u, v, w)}{\Psi(x, u, \lambda)}
$$

subject to (27), (30)-(35), and

$$
\begin{gathered}
\Psi(x, u, \lambda)\left\{\sum_{i=1}^{r} \lambda_{i}\left[\nabla_{1} f_{i}(x, u, t)+K_{i}(t)^{T} \alpha^{i}(t)\right]+A(t)^{T} v(t)\right. \\
\left.+\sum_{j=1}^{s} w_{j}(t)\left[\nabla_{1} h_{j}(x, u, t)+P_{j}(t)^{T} \zeta^{j}(t)\right]+D v(t)\right\} \\
-[\Phi(x, u, \lambda)+\Omega(x, u, v, w)] \sum_{i=1}^{r} \lambda_{i}\left[\nabla_{1} g_{i}(x, u, t)\right. \\
\left.-M_{i}(t)^{T} \gamma^{i}(t)\right]=0, \quad t \in[a, b], \\
\Psi(x, u, \lambda)\left\{\sum_{i=1}^{r} \lambda_{i}\left\{\nabla_{2} f_{i}(x, u, t)+L_{i}(t)^{T} \beta^{i}(t)\right]+B(t)^{T} v(t)\right. \\
\left.+\sum_{j=1}^{s} w_{j}(t)\left[\nabla_{2} h_{j}(x, u, t)+Q_{j}(t)^{T} \eta^{j}(t)\right]\right\}
\end{gathered}
$$




$$
\begin{aligned}
-[\Phi(x, u, \lambda)+\Omega(x, u, v, w)] \sum_{i=1}^{r} \lambda_{i}\left[\nabla_{2} g_{i}(x, u, t)\right. & \\
& \left.-N_{i}(t)^{T} \delta^{i}(t)\right]=0, \quad t \in[a, b],
\end{aligned}
$$

where $\Phi$ and $\Psi$ are as defined in Theorem 3.4 and

$$
\begin{aligned}
\Omega(x, u, v, w)= & \int_{a}^{b}\left\{v(t)^{T}[-D x-A(t) x+B(t) u]\right. \\
& +\sum_{j=1}^{s} w_{j}(t)\left[h_{j}(x, u, t)+\left\|P_{j}(t) x\right\|_{p(j)}\right. \\
& \left.\left.+\left\|Q_{j}(t) u\right\|_{q(j)}\right]\right\} d t
\end{aligned}
$$

$$
(\tilde{D} I I I) \quad \text { Maximize } \frac{\Phi(x, u, \lambda)+\Omega(x, u, v, w)}{\Psi(x, u, \lambda)}
$$

subject to $(27),(30)-(35)$, and

$$
\begin{aligned}
\{\Psi(x, u, \lambda) & \left\{\sum_{i=1}^{r} \lambda_{i}\left[\nabla_{1} f_{i}(x, u, t)^{T}+\alpha^{i}(t)^{T} K_{i}(t)\right]+v(t)^{T} A(t)\right. \\
+ & \left.\sum_{j=1}^{s} w_{j}(t)\left[\nabla_{1} h_{j}(x, u, t)^{T}+\zeta^{j}(t)^{T} P_{j}(t)\right]+D v(t)^{T}\right\} \\
& -[\Phi(x, u, \lambda)+\Omega(x, u, v, w)] \sum_{i=1}^{r} \lambda_{i}\left[\nabla_{1} g_{i}(x, u, t)^{T}\right. \\
& \left.\left.-\gamma^{i}(t)^{T} M_{i}(t)\right]\right\}(\bar{x}-x) \geq 0
\end{aligned}
$$

for all $t \in[a, b]$ and $\bar{x} \in C^{n}[a, b]$ such that $(\bar{x}, u) \in \mathbb{F}$ for some $u \in$ $P W S^{m}[a, b]$,

$$
\begin{aligned}
\{\Psi(x, u, \lambda)\{ & \sum_{i=1}^{r} \lambda_{i}\left[\nabla_{2} f_{i}(x, u, t)^{T}+\beta^{i}(t)^{T} L_{i}(t)\right]+v(t)^{T} B(t) \\
& \left.+\sum_{j=1}^{s} w_{j}(t)\left[\nabla_{2} h_{j}(x, u, t)^{T}+\eta^{j}(t)^{T} Q_{j}(t)\right]\right\} \\
- & {[\Phi(x, u, \lambda)+\Omega(x, u, v, w)] \sum_{i=1}^{r} \lambda_{i}\left[\nabla_{2} g_{i}(x, u, t)^{T}\right.} \\
& \left.\left.-\delta^{i}(t)^{T} N_{i}(t)\right]\right\}(\bar{u}-u) \geq 0
\end{aligned}
$$


for all $t \in[a, b]$ and $\bar{u} \in P W S^{m}[a, b]$ such that $(x, \bar{u}) \in \mathbb{F}$ for some $x \in C^{n}[a, b]$.

The remarks made earlier about the relationships between (DI) and $(\tilde{D} \mathrm{I})$ are, of course, also applicable to (DIII) and ( $\tilde{D} \mathrm{III})$.

We next state and prove weak, strong, and strict converse duality theorems for (P) and (DIII).

Theorem 6.1. (Weak Duality) Let $(\bar{x}, \bar{u})$ and $z \equiv(x, u, \lambda, v, w, \alpha, \beta$, $\gamma, \delta, \zeta, \eta)$ be arbitrary feasible solutions of $(P)$ and $(D I I I)$, respectively. Then $\varphi(\bar{x}, \bar{u}) \geq \xi(z)$, where $\xi$ is the objective function of (DIII).

Proof. Keeping in mind that $\lambda \geq 0, \Phi(x, u, \lambda) \geq 0, \Psi(x, u, \lambda)>0$, and $w(t) \geq 0$ for all $t \in[a, b]$, we have

$$
\begin{aligned}
& \Psi(x, u, \lambda) \Phi(\bar{x}, \bar{u}, \lambda)-[\Phi(x, u, \lambda)+\Omega(x, u, v, w)] \Psi(\bar{x}, \bar{u}, \lambda) \\
= & \Psi(x, u, \lambda) \int_{a}^{b} \sum_{i=1}^{r} \lambda_{i}\left[f_{i}(\bar{x}, \bar{u}, t)+\left\|K_{i}(t) \bar{x}\right\|_{k(i)}+\left\|L_{i}(t) \bar{u}\right\|_{\ell(i)}\right] \\
& \left.-f_{i}(x, u, t)-\left\|K_{i}(t) x\right\|_{k(i)}-\left\|L_{i}(t) u\right\|_{\ell(i)}\right] d t \\
& -\Phi(x, u, \lambda) \int_{a}^{b} \sum_{i=1}^{r} \lambda_{i}\left[g_{i}(\bar{x}, \bar{u}, t)-\left\|M_{i}(t) \bar{x}\right\|_{m(i)}-\left\|N_{i}(t) \bar{u}\right\|_{n(i)}\right. \\
& \left.-g_{i}(x, u, t)+\left\|M_{i}(t) x\right\|_{m(i)}+\left\|N_{i}(t) u\right\|_{n(i)}\right] d t-\Psi(\bar{x}, \bar{u}, \lambda) \Omega(x, u, v, w) \\
\geq & \Psi(x, u, \lambda) \int_{a}^{b} \sum_{i=1}^{r} \lambda_{i}\left[\nabla_{1} f_{i}(x, u, t)^{T}(\bar{x}-x)+\nabla_{2} f_{i}(x, u, t)^{T}(\bar{u}-u)\right. \\
& \left.+\left\|K_{i}(t) \bar{x}\right\|_{k(i)}+\left\|L_{i}(t) \bar{u}\right\|_{\ell(i)}-\left\|K_{i}(t) x\right\|_{k(i)}-\left\|L_{i}(t) u\right\|_{\ell(i)}\right] d t \\
& -\Phi(x, u, \lambda) \int_{a}^{b} \sum_{i=1}^{r} \lambda_{i}\left[\nabla_{1} g_{i}(x, u, t)^{T}(\bar{x}-x)+\nabla_{2} g_{i}(x, u, t)^{T}(\bar{u}-u)\right. \\
& \left.-\left\|M_{i}(t) \bar{x}\right\|_{m(i)}-\left\|N_{i}(t) \bar{u}\right\|_{n(i)}+\left\|M_{i}(t) x\right\|_{m(i)}+\left\|N_{i}(t) u\right\|_{n(i)}\right] d t \\
& -\Psi(\bar{x}, \bar{u}, \lambda) \Omega(x, u, v, w) \\
& \left(\text { by the convexity of } f_{i}(., ., t) \text { and }-g_{i}(., ., t), i \in \underline{r}\right)
\end{aligned}
$$$$
=\int_{a}^{b}\left\{-\Psi(x, u, \lambda)\left\{\sum _ { i = 1 } ^ { r } \lambda _ { i } \left[\alpha^{i}(t)^{T} K_{i}(t)(\bar{x}-x)+\beta^{i}(t)^{T} L_{i}(t)(\bar{u}-u)\right.\right.\right.
$$$$
\left.-\left\|K_{i}(t) \bar{x}\right\|_{k(i)}-\left\|L_{i}(t) \bar{u}\right\|_{\ell(i)}+\left\|K_{i}(t) x\right\|_{k(i)}+\left\|L_{i}(t) u\right\|_{\ell(i)}\right]
$$$$
+\sum_{j=1}^{s} w_{j}(t)\left\{\left[\nabla_{1} h_{j}(x, u, t)^{T}+\zeta^{j}(t)^{T} P_{j}(t)\right](\bar{x}-x)+\left[\nabla_{2} h_{j}(x, u, t)^{T}\right.\right.
$$ 


$$
\begin{aligned}
& \left.\left.+\eta^{j}(t)^{T} Q_{j}(t)\right](\bar{u}-u)\right\}+\left[v(t)^{T} A(t)+D v(t)^{T}\right](\bar{x}-x) \\
& \left.+v(t)^{T} B(t)(\bar{u}-u)\right\} \\
& +\Phi(x, u, \lambda) \sum_{i=1}^{r} \lambda_{i}\left[-\gamma^{i}(t)^{T} M_{i}(t)(\bar{x}-x)-\delta^{i}(t)^{T} N_{i}(t)(\bar{u}-u)\right. \\
& \left.-\left\|M_{i}(t) x\right\|_{m(i)}-\left\|N_{i}(t) u\right\|_{n(i)}+\left\|M_{i}(t) \bar{x}\right\|_{m(i)}+\left\|N_{i}(t) \bar{u}\right\|_{n(i)}\right] \\
& +\Omega(x, u, v, w) \sum_{i=1}^{r} \lambda_{i}\left\{\nabla_{1} g_{i}(x, u, t)^{T}(\bar{x}-x)+\left[\nabla_{2} g_{i}(x, u, t)^{T}(\bar{u}-u)\right]\right. \\
& \left.\left.\left.-\gamma^{i}(t)^{T} M_{i}(t)(\bar{x}-x)-\delta^{i}(t)^{T} N_{i}(t)(\bar{u}-u)\right]\right\}\right\} d t \\
& -\Phi(\bar{x}, \bar{u}, \lambda) \Omega(x, u, v, w) \text { (by (41) and (42)) } \\
& \geq \int_{a}^{b}\left\{\Psi ( x , u , \lambda ) \left\{\sum _ { i = 1 } ^ { r } \lambda _ { i } \left[-\left\|K_{i}(t) \bar{x}\right\|_{k(i)}\left\|\alpha^{i}(t)\right\|_{k(i)}^{*}+\alpha^{i}(t)^{T} K_{i}(t) x\right.\right.\right. \\
& +\left\|L_{i}(t) \bar{u}\right\|_{\ell(i)}\left\|\beta^{i}(t)\right\|_{\ell(i)}^{*}+\beta^{i}(t)^{T} L_{i}(t) u+\left\|K_{i}(t) \bar{x}\right\|_{k(i)} \\
& \left.+\left\|L_{i}(t) \bar{u}\right\|_{\ell(i)}-\left\|K_{i}(t) x\right\|_{k(i)}-\left\|L_{i}(t) u\right\|_{\ell(i)}\right] \\
& -\sum_{j=1}^{s} w_{j}(t)\left[\nabla_{1} h_{j}(x, u, t)^{T}(\bar{x}-x)\right. \\
& +\left\|P_{j}(t) \bar{x}\right\|_{p(j)}\left\|\zeta^{j}(t)\right\|_{p(j)}^{*}-\zeta^{j}(t)^{T} P_{j}(t) x+\nabla_{2} h_{j}(x, u, t)^{T}(\bar{u}-u) \\
& \left.+\left\|Q_{j}(t) \bar{u}\right\|_{q(j)}\left\|\eta^{j}(t)\right\|_{q(j)}^{*}-\eta^{j}(t)^{T} Q_{j}(t) u\right] \\
& \left.+v(t)^{T}[D \bar{x}-A(t) \bar{x}-B(t) \bar{u}]+v(t)^{T}[-D x+A(t) x+B(t) u]\right\} \\
& +\Phi(x, u, \lambda) \sum_{i=1}^{r} \lambda_{i}\left[-\left\|M_{i}(t) \bar{x}\right\|_{m(i)}\left\|\gamma^{i}(t)\right\|_{m(i)}^{*}+\gamma^{i}(t)^{T} M_{i}(t) x\right. \\
& -\left\|N_{i}(t) \bar{u}\right\|_{n(i)}\left\|\delta^{i}(t)\right\|_{n(i)}^{*}+\delta^{i}(t)^{T} N_{i}(t) u-\left\|M_{i}(t) x\right\|_{m(i)} \\
& \left.-\left\|N_{i}(t) u\right\|_{n(i)}+\left\|M_{i}(t) \bar{x}\right\|_{m(i)}+\left\|N_{i}(t) \bar{u}\right\|_{n(i)}\right] \\
& +\Omega(x, u, v, w) \sum_{i=1}^{r} \lambda_{i}\left[-g_{i}(x, u, t)+g_{i}(\bar{x}, \bar{u}, t)\right. \\
& -\left\|M_{i}(t) \bar{x}\right\|_{m(i)}\left\|\gamma^{i}(t)\right\|_{m(i)}^{*}+\gamma^{i}(t)^{T} M_{i}(t) x \\
& \text { - }\left\|N_{i}(t) \bar{u}\right\|_{n(i)}\left\|\delta^{i}(t)\right\|_{n(i)}^{*}+\delta^{i}(t)^{T} N_{i}(t) u \\
& \left.\left.-g_{i}(\bar{x}, \bar{u}, t)+\left\|M_{i}(t) \bar{x}\right\|_{m(i)}+\left\|N_{i}(t) \bar{u}\right\|_{n(i)}\right]\right\} d t
\end{aligned}
$$


(by Lemma 3.2, integration by parts,

convexity of $-g_{i}(., ., t), i \in \underline{r}$, and definition of $\left.\Psi(\bar{x}, \bar{u}, \lambda)\right)$

$\geq \int_{a}^{b}\left\{\Psi(x, u, \lambda)\left\{v(t)^{T}[-D x+A(t) x+B(t) u]\right.\right.$

$+\sum_{j=1}^{s} w_{j}(t)\left[h_{j}(x, u, t)-h_{j}(\bar{x}, \bar{u}, t)-\left\|P_{j}(t) \bar{x}\right\|_{p(j)}\right.$

$\left.\left.-\left\|Q_{j}(t) \bar{u}\right\|_{q(j)}+\zeta^{j}(t)^{T} P_{j}(t) x+\eta^{j}(t)^{T} Q_{j}(t) u\right]\right\}$

$\left.-\Omega(x, u, v, w) \sum_{i=1}^{r} \lambda_{i}\left[g_{i}(x, u, t)-\gamma^{i}(t)^{T} M_{i}(t) x-\delta^{i}(t)^{T} N_{i}(t) u\right]\right\} d t$

(by $(31)-(33)$, primal feasibility of $(\bar{x}, \bar{u})$,

and convexity of $\left.h_{j}(., ., t), j \in \underline{s}\right)$

$\geq 0$ (by the primal feasibility of $(\bar{x}, \bar{u}),(33),(34)$,

and definitions of $\Psi(x, u, \lambda)$ and $\Omega(x, u, v, w))$.

From the above inequality we conclude that

$$
\frac{\Phi(\bar{x}, \bar{u}, \lambda)}{\Psi(\bar{x}, \bar{u}, \lambda)} \geq \frac{\Phi(x, u, \lambda)+\Omega(x, u, v, w)}{\Psi(x, u, \lambda)} .
$$

Because

$$
\varphi(\bar{x}, \bar{u})=\max _{\mu \in \Lambda} \frac{\Phi(\bar{x}, \bar{u}, \mu)}{\Psi(\bar{x}, \bar{u}, \mu)}
$$

by Lemma 3.3 , it follows from (43) that

$$
\varphi(\bar{x}, \bar{u}) \geq \frac{\Phi(x, u, \lambda)+\Omega(x, u, v, w)}{\Psi(x, u, \lambda)}=\xi(z) .
$$

THEOREM 6.2. (Strong Duality) Let $\left(x^{*}, u^{*}\right)$ be an optimal solution of $(P)$. Then there exist $\lambda^{*} \in \Lambda, v^{*} \in P W S^{n}[a, b], w^{*} \in P W S_{+}^{s}[a, b], \alpha^{* i} \in$ $P W S^{k_{i}}[a, b], \beta^{* i} \in P W S^{\ell_{i}}[a, b], \gamma^{* i} \in P W S^{m_{i}}[a, b], \delta^{* i} \in P W S^{n_{i}}[a, b]$, $i \in \underline{r}, \zeta^{* j} \in P W S^{p_{j}}[a, b], \eta^{* j} \in P W S^{q_{j}}[a, b], j \in \underline{s}$, such that $z^{*} \equiv$ $\left(x^{*}, u^{*}, \lambda^{*}, v^{*}, w^{*}, \alpha^{*}, \beta^{*}, \gamma^{*}, \delta^{*}, \zeta^{*}, \eta^{*}\right)$ is an optimal solution of (DIII) and $\varphi\left(x^{*}, u^{*}\right)=\xi\left(z^{*}\right)$.

Proof. By Theorem 3.4, there exist $v^{\circ} \in P W S^{n}[a, b], w^{\circ} \in P W S^{s}[a, b]$, and $\lambda^{*}, \alpha^{* i}, \beta^{* i}, \gamma^{* i}, \delta^{* i}, i \in \underline{r}, \zeta^{* j}, \eta^{* j}, j \in \underline{s}$, as specified above, such 
that the following relations hold for all $t \in[a, b]$ :

(44)

$$
\begin{aligned}
& \sum_{i=1}^{r} \lambda_{i}^{*}\left\{\Psi\left(x^{*}, u^{*}, \lambda^{*}\right)\left[\nabla_{1} f_{i}\left(x^{*}, u^{*}, t\right)+K_{i}(t)^{T} \alpha^{* i}(t)\right]\right. \\
& \left.-\Phi\left(x^{*}, u^{*}, \lambda^{*}\right)\left[\nabla_{1} g_{i}\left(x^{*}, u^{*}, t\right)-M_{i}(t)^{T} \gamma^{* i}(t)\right]\right\}+A(t)^{T} v^{\circ}(t) \\
& \quad+\sum_{j=1}^{s} w_{j}^{\circ}(t)\left[\nabla_{1} h_{j}\left(x^{*}, u^{*}, t\right)+P_{j}(t)^{T} \zeta^{* j}(t)\right]+D v^{\circ}(t)=0
\end{aligned}
$$

(45) $\sum_{i=1}^{r} \lambda_{i}^{*}\left\{\Psi\left(x^{*}, u^{*}, \lambda^{*}\right)\left[\nabla_{2} f_{i}\left(x^{*}, u^{*}, t\right)+L_{i}(t)^{T} \beta^{* i}(t)\right]\right.$

$$
\begin{aligned}
-\Phi\left(x^{*}, u^{*}, \lambda^{*}\right) & {\left.\left[\nabla_{2} g_{i}\left(x^{*}, u^{*}, t\right)-N_{i}(t)^{T} \delta^{* i}(t)\right]\right\}+B(t)^{T} v^{\circ}(t) } \\
& +\sum_{j=1}^{s} w_{j}^{\circ}(t)\left[\nabla_{2} h_{j}\left(x^{*}, u^{*}, t\right)+Q_{j}(t)^{T} \eta^{* j}(t)\right]=0,
\end{aligned}
$$

(46) $\quad \sum_{j=1}^{s} w_{j}^{\circ}(t)\left[h_{j}\left(x^{*}, u^{*}, t\right)+\left\|P_{j}(t) x^{*}\right\|_{p(j)}+\left\|Q_{j}(t) u^{*}\right\|_{q(j)}\right]=0$

$$
\varphi\left(x^{*}, u^{*}\right)=\frac{\Phi\left(x^{*}, u^{*}, \lambda^{*}\right)}{\Psi\left(x^{*}, u^{*}, \lambda^{*}\right)}
$$

(48) $\left\|\alpha^{* i}(t)\right\|_{k(i)}^{*} \leq 1, \quad\left\|\beta^{* i}(t)\right\|_{\ell(i)}^{*} \leq 1$

$$
\left\|\gamma^{* i}(t)\right\|_{m(i)}^{*} \leq 1,\left\|\delta^{* i}(t)\right\|_{n(i)}^{*} \leq 1, \quad i \in \underline{r},
$$

$$
\left\|\zeta^{* j}(t)\right\|_{p(j)}^{*} \leq 1, \quad\left\|\eta^{* j}(t)\right\|_{q(j)}^{*} \leq 1, j \in \underline{s}
$$

(50) $\quad \alpha^{* i}(t)^{T} K_{i}(t) x^{*}=\left\|K_{i}(t) x^{*}\right\|_{k(i)}, \quad \beta^{* i}(t)^{T} L_{i}(t) u^{*}=\left\|L_{i}(t) u^{*}\right\|_{\ell(i)}$,

$$
\begin{aligned}
& \gamma^{* i}(t)^{T} M_{i}(t) x^{*}=\left\|M_{i}(t) x^{*}\right\|_{m(i)}, \quad \delta^{* i}(t)^{T} N_{i}(t) u^{*}=\left\|N_{i}(t) u^{*}\right\|_{n(i)}, \\
& i \in \underline{r},
\end{aligned}
$$

$\zeta^{* j}(t)^{T} P_{j}(t) x^{*}=\left\|P_{j}(t) x^{*}\right\|_{p(j)}, \quad \eta^{* j}(t)^{T} Q_{j}(t) u^{*}=\left\|Q_{j}(t) u^{*}\right\|_{q(j)}, \quad j \in \underline{s}$. 
Since $\Psi\left(x^{*}, u^{*}, \lambda^{*}\right)>0$ and (46) holds, (44)-(46) can be rewritten as follows :

$$
\begin{gathered}
\Psi\left(x^{*}, u^{*}, \lambda^{*}\right)\left\{\sum_{i=1}^{r} \lambda_{i}^{*}\left[\nabla_{1} f_{i}\left(x^{*}, u^{*}, t\right)+K_{i}(t)^{T} \alpha^{* i}(t)\right]\right. \\
+A(t)^{T}\left[v^{\circ} / \Psi\left(x^{*}, u^{*}, \lambda^{*}\right)\right]+\sum_{j=1}^{s}\left[w_{j}^{\circ}(t) / \Psi\left(x^{*}, u^{*}, \lambda^{*}\right)\right] \times \\
{\left[\nabla_{1} h_{j}\left(x^{*}, u^{*}, t\right)+P_{j}(t)^{T} \zeta^{* j}(t)\right]} \\
\left.+D\left[v^{\circ}(t) / \Psi\left(x^{*}, u^{*}, \lambda^{*}\right)\right]\right\}-\left[\Phi\left(x^{*}, u^{*}, \lambda^{*}\right)\right. \\
\left.+\Omega\left(x^{*}, u^{*}, v^{\circ} / \Psi\left(x^{*}, u^{*}, \lambda^{*}\right), w^{\circ} / \Psi\left(x^{*}, u^{*}, \lambda^{*}\right)\right)\right] \sum_{i=1}^{r} \lambda_{i}^{*}\left[\nabla_{1} g_{i}\left(x^{*}, u^{*}, t\right)\right. \\
\left.-M_{i}(t)^{T} \gamma^{* i}(t)\right]=0, \quad t \in[a, b],
\end{gathered}
$$

$$
\begin{aligned}
& \Psi\left(x^{*}, u^{*}, \lambda^{*}\right)\left\{\sum_{i=1}^{r} \lambda_{i}^{*}\left[\nabla_{2} f_{i}\left(x^{*}, u^{*}, t\right)+L_{i}(t)^{T} \beta^{* i}(t)\right]\right. \\
& +B(t)^{T}\left[v^{\circ} / \Psi\left(x^{*}, u^{*}, \lambda^{*}\right)\right]+\sum_{j=1}^{s}\left[w_{j}^{\circ}(t) / \Psi\left(x^{*}, u^{*}, \lambda^{*}\right)\right] \times \\
& \left.\left[\nabla_{2} h_{j}\left(x^{*}, u^{*}, t\right)+Q_{j}(t)^{T} \eta^{* j}(t)\right]\right\}-\left[\Phi\left(x^{*}, u^{*}, \lambda^{*}\right)\right. \\
& \left.+\Omega\left(x^{*}, u^{*}, v^{\circ} / \Psi\left(x^{*}, u^{*}, \lambda^{*}\right), w^{\circ} / \Psi\left(x^{*}, u^{*}, \lambda^{*}\right)\right)\right] \times \\
& \sum_{i=1}^{r} \lambda_{i}^{*}\left[\nabla_{2} g_{i}\left(x^{*}, u^{*}, t\right)-N_{i}(t)^{T} \delta^{* i}(t)\right]=0, \quad t \in[a, b],
\end{aligned}
$$

$$
\begin{aligned}
& \sum_{j=1}^{s}\left[w_{j}^{\circ}(t) / \Psi\left(x^{*}, u^{*}, \lambda^{*}\right)\right] \times \\
& \quad\left[h_{j}\left(x^{*}, u^{*}, t\right)+\left\|P_{j}(t) x^{*}\right\|_{p(j)}+\left\|Q_{j}(t) u^{*}\right\|_{q(j)}\right]=0, \quad t \in[a, b] .
\end{aligned}
$$

Now letting $v^{*}=v^{\circ} / \Psi\left(x^{*}, u^{*}, \lambda^{*}\right)$ and $w^{*}=w^{\circ} / \Psi\left(x^{*}, u^{*}, \lambda^{*}\right)$ in (44)(46), we see from (48)-(54) that $z^{*}$ is a feasible solution of (DIII). But in view of (47) and (54), $\varphi\left(x^{*}, u^{*}\right)=\xi\left(z^{*}\right)$ and, therefore, by Theorem $6.1,\left(x^{*}, u^{*}\right)$ is an optimal solution of (DIII). 
TheOREM 6.3. (Strict Converse Duality) Let $\left(x^{*}, u^{*}\right)$ and $\tilde{z} \equiv(\tilde{x}, \tilde{u}$, $\tilde{\lambda}, \tilde{v}, \tilde{w}, \tilde{\alpha}, \tilde{\beta}, \tilde{\gamma}, \tilde{\delta}, \tilde{\zeta}, \tilde{\eta})$ be optimal solutions of $(P)$ and (DIII), respectively, and assume that $f_{i}(., ., t)$ or $\rightarrow g_{i}(., ., t)$ is strictly convex throughout $[a, b]$ for at least one index $i \in \underline{r}$ with the corresponding component $\tilde{\lambda}_{i}$ of $\tilde{\lambda}$ (and $\Phi(\tilde{x}, \tilde{u}, \tilde{\lambda})$ ) positive, or $h_{j}(., ., t)$ is strictly convex throughout $[a, b]$ for at least one $j \in \underline{s}$ with the corresponding component $\tilde{w}_{j}(t)$ of $\tilde{w}(t)$ positive on $[a, b]$. Then $(\tilde{x}(t), \tilde{u}(t))=\left(x^{*}(t), u^{*}(t)\right)$ for each $t \in[a, b]$, that is, $(\tilde{x}, \tilde{u})$ is an optimal solution of $(P)$, and $\varphi\left(x^{*}, u^{*}\right)=\xi(\tilde{z})$.

Proof. The proof is similar to that of Theorem 5.3.

We close this section with a brief discussion of the reduced versions of (DIII) and ( $\tilde{D}$ III) obtained by omitting (33) and (34) and modifying the objective functions and the remaining constraints of (DIII) and ( $\tilde{D}$ III) accordingly. These problems assume the following forms :

$$
(D I I I A) \quad \text { Maximize } \frac{\Pi(x, u, \lambda, \alpha, \beta)+\Delta(x, u, v, w, \zeta, \eta)}{\Gamma(x, u, \lambda, \gamma, \delta)}
$$

subject to

$$
\begin{gathered}
x(a)=0, \quad x(b)=0 \\
\Gamma(x, u, \lambda, \gamma, \delta)\left\{\sum_{i=1}^{r} \lambda_{i}\left[\nabla_{1} f_{i}(x, u, t)+K_{i}(t)^{T} \alpha^{i}(t)\right] A(t)^{T} v(t)\right. \\
\left.+\sum_{j=1}^{s} w_{j}(t)\left[\nabla_{1} h_{j}(x, u, t)+P_{j}(t)^{T} \zeta^{j}(t)\right]+D v(t)\right\} \\
-[\Pi(x, u, \lambda, \alpha, \beta)+\Delta(x, u, v, w, \zeta, \eta)] \times \\
\sum_{i=1}^{r} \lambda_{i}\left[\nabla_{1} g_{i}(x, u, t)-M_{i}(t)^{T} \gamma^{i}(t)\right]=0, \quad t \in[a, b]
\end{gathered}
$$

$$
\begin{aligned}
& \Gamma(x, u, \lambda, \gamma, \delta)\left\{\sum_{i=1}^{r} \lambda_{i}\left[\nabla_{2} f_{i}(x, u, t)+L_{i}(t)^{T} \beta^{i}(t)\right]\right. \\
&\left.+B(t)^{T} v(t)+\sum_{j=1}^{s} w_{j}(t)\left[\nabla_{2} h_{j}(x, u, t)+Q_{j}(t)^{T} \eta^{j}(t)\right]\right\}
\end{aligned}
$$


$-[\Pi(x, u, \lambda, \alpha, \beta)+\Delta(x, u, v, w, \zeta, \eta)] \times$

$$
\sum_{i=1}^{r} \lambda_{i}\left[\nabla_{2} g_{i}(x, u, t)-N_{i}(t)^{T} \delta^{i}(t)\right]=0, \quad t \in[a, b],
$$

(58) $v(t)^{T}[-D x-A(t) x+B(t) u]+\sum_{j=1}^{s} w_{j}(t)\left[h_{j}(x, u, t)\right.$

$$
\left.+\left\|P_{j}(t) x\right\|_{p(j)}+\left\|Q_{j}(t) u\right\|_{q(j)}\right] \geq 0, \quad t \in[a, b],
$$

(59) $\left\|\alpha^{i}(t)\right\|_{k(i)}^{*} \leq 1, \quad\left\|\beta^{i}(t)\right\|_{\ell(i)}^{*} \leq 1, \quad\left\|\gamma^{i}(t)\right\|_{m(i)}^{*} \leq 1$,

$$
\left\|\delta^{i}(t)\right\|_{n(i)}^{*} \leq 1, \quad t \in[a, b], \quad i \in \underline{r},
$$

$$
\left\|\zeta^{j}(t)\right\|_{p(j)}^{*} \leq 1, \quad\left\|\eta^{j}(t)\right\|_{q(j)}^{*} \leq 1, \quad t \in[a, b], \quad j \in \underline{s},
$$

(61) $\lambda \in \Lambda, v \in P W S^{n}[a, b], w \in P W S_{+}^{s}[a, b], \alpha^{i} \in P W S^{k_{i}}[a, b]$,

$$
\begin{aligned}
\beta^{i} \in P W S^{\ell_{i}}[a, b], \gamma^{i} & \in P W S^{m_{i}}[a, b], \delta^{i} \in P W S^{n_{i}}[a, b], \\
i \in \underline{r} ; \zeta^{j} & \in P W S^{p_{j}}[a, b], \eta^{j} \in P W S^{q_{j}}[a, b], j \in \underline{s},
\end{aligned}
$$

where $\Pi$ and $\Gamma$ are as defined in the preceding section and

$$
\begin{aligned}
& \Delta(x, u, v, w, \zeta, \eta)=\int_{a}^{b}\left\{v(t)^{T}[-D x-A(t) x-B(t) u]\right. \\
& \left.+\sum_{j=1}^{s} w_{j}(t)\left[h_{j}(x, u, t)+\zeta^{j}(t)^{T} P_{j}(t) x+\eta^{j}(t)^{T} Q_{j}(t) u\right]\right\} d t
\end{aligned}
$$

$(\tilde{D} I I I A) \quad$ Maximize $\frac{\Pi(x, u, \lambda, \alpha, \beta)+\Delta(x, u, v, w, \zeta, \eta)}{\Gamma(x, u, \lambda, \gamma, \delta)}$

subject to (55), (58)-(61), and

$$
\begin{aligned}
\left\{\Gamma ( x , u , \lambda , \gamma , \delta ) \left\{\sum_{i=1}^{r} \lambda_{i}\left[\nabla_{1} f_{i}(x, u, t)^{T}+\alpha^{i}(t)^{T} K_{i}(t)\right]+v(t)^{T} A(t)\right.\right. \\
\left.+\sum_{j=1}^{s} w_{j}(t)\left[\nabla_{1} h_{j}(x, u, t)^{T}+\zeta^{j}(t)^{T} P_{j}(t)\right]+D v(t)^{T}\right\} \\
-[\Pi(x, u, \lambda, \alpha, \beta)+\Delta(x, u, v, w, \zeta, \eta)] \times \\
\left.\sum_{i=1}^{r} \lambda_{i}\left[\nabla_{1} g_{i}(x, u, t)^{T}-\gamma^{i}(t)^{T} M_{i}(t)\right]\right\}(\bar{x}-x) \geq 0
\end{aligned}
$$


for all $t \in[a, b]$ and $\bar{x} \in C^{n}[a, b]$ such that $(\bar{x}, u) \in \mathbb{F}$ for some $u \in$ $P W S^{m}[a, b]$

$$
\begin{gathered}
\left\{\Gamma ( x , u , \lambda , \gamma , \delta ) \left\{\sum_{i=1}^{r} \lambda_{i}\left[\nabla_{2} f_{i}(x, u, t)^{T}+\beta^{i}(t)^{T} L_{i}(t)\right]+v(t)^{T} B(t)\right.\right. \\
\left.+\sum_{j=1}^{s} w_{j}(t)\left[\nabla_{2} h_{j}(x, u, t)^{T}+\eta^{j}(t)^{T} Q_{j}(t)\right]\right\} \\
-[\Pi(x, u, \lambda, \alpha, \beta)+\Delta(x, u, v, w, \zeta, \eta)] \times \\
\left.\sum_{i=1}^{r} \lambda_{i}\left[\nabla_{2} g_{i}(x, u, t)^{T}-\delta^{i}(t)^{T} N_{i}(t)\right]\right\}(\bar{u}-u) \geq 0
\end{gathered}
$$

for all $t \in[a, b]$ and $\bar{u} \in P W S^{m}[a, b]$ such that $(x, \bar{u}) \in \mathbb{F}$ for some $x \in C^{n}[a, b]$.

The remarks made earlier about the relationships between (DI) and

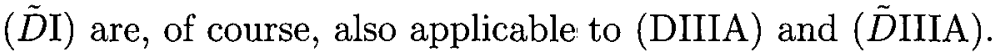

Following the pattern of Theorems 5.4-5.6 and Theorems 6.1-6.3, one can state and prove similar duality theorems for (P)-(DIIIA) (and (P)( $\tilde{D}$ IIIA)).

\section{Problems containing square roots of positive semidefinite quadratic forms}

In this section, we briefly discuss an important special case of $(\mathrm{P})$ obtained by choosing all the norms to be the $\ell_{2}$-norm $\|\cdot\|_{2}$. Specifically, if we let $k(i)=\ell(i)=m(i)=n(i)=p(j)=q(j)=2$ and define $E_{i}(t)=K_{i}(t)^{T} K_{i}(t), F_{i}(t)=L_{i}(t)^{T} L_{i}(t), G_{i}(t)=M_{i}(t)^{T} M_{i}(t), H_{i}(t)=$ $N_{i}(t)^{T} N_{i}(t), i \in \underline{r}, R_{j}(t)=P_{j}(t)^{T} P_{j}(t)$ and $S_{j}(t)=Q_{j}(t)^{T} Q_{j}(t), j \in$ $\underline{s}$, then it is easily seen that $E_{i}(t), G_{i}(t), i \in \underline{r}$, and $R_{j}(t), j \in \underline{s}$, are $n \times n$ symmetric positive semidefinite matrices; $F_{i}(t), H_{i}(t), i \in \underline{r}$, and $S_{j}(t), j \in \underline{s}$, are $m \times m$ symmetric positive semidefinite matrices, and, therefore, the functions $x(t) \rightarrow\left[x(t)^{T} E_{i}(t) x(t)\right]^{1 / 2}, x(t) \rightarrow$ $\left[x(t)^{T} G_{i}(t) x(t)\right]^{1 / 2}, \quad i \in \underline{r}$, and $x(t) t\left[x(t)^{T} R_{j}(t) x(t)\right]^{1 / 2}, j \in \underline{s}$, are convex on $\mathbb{R}^{n}$, and the functions $u(t) \rightarrow\left[u(t)^{T} F_{i}(t) u(t)\right]^{1 / 2}, u(t) \rightarrow$ $\left[u(t)^{T} H_{i}(t) u(t)\right]^{1 / 2}, i \in \underline{r}$, and $u(t) \rightarrow\left[u(t)^{T} S_{j}(t) u(t)\right]^{1 / 2}, j \in \underline{s}$, are convex on $\mathbb{R}^{m}$. With these choices of the norms and matrices, (P), (P1), (P2), and (P3) become

$$
\left(P^{*}\right) \text { Minimize } \max _{1 \leq i \leq r} \frac{\int_{a}^{b}\left\{f_{i}(x(t), u(t), t)+\left[x(t)^{T} E_{i}(t) x(t)\right]^{1 / 2}+\left[u(t)^{T} F_{i}(t) u(t)\right]^{1 / 2}\right\} d t}{\int_{a}^{b}\left\{g_{i}(x(t), u(t), t)-\left[x(t)^{T} G_{i}(t) x(t)\right]^{1 / 2}-\left[u(t)^{T} H_{i}(t) u(t)\right]^{1 / 2}\right\} d t}
$$


subject to

$$
\begin{gathered}
x(a)=0, \quad x(b)=0, \\
D x(t)=A(t) x(t)+B(t) u(t), \quad t \in[a, b],
\end{gathered}
$$

$h_{j}(x(t), u(t), t)+\left[x(t)^{T} R_{j}(t) x(t)\right]^{1 / 2}$

$$
+\left[u(t)^{T} S_{j}(t) u(t)\right]^{1 / 2} \leq 0, \quad t \in[a, b], \quad j \in \underline{s},
$$

$\left(P 1^{*}\right) \quad \underset{(x, u) \in \mathbb{F}^{*}}{\operatorname{Minimize}} \frac{\int_{a}^{b}\left\{f_{1}(x(t), u(t), t)+\left[x(t)^{T} E_{1}(t) x(t)\right]^{1 / 2}+\left[u(t)^{T} F_{1}(t) u(t)\right]^{1 / 2}\right\} d t}{\int_{a}^{b}\left\{g_{1}(x(t), u(t), t)-\left[x(t)^{T} G_{1}(t) x(t)\right]^{1 / 2}-\left[u(t)^{T} H_{1}(t) u(t)\right]^{1 / 2}\right\} d t} ;$

$\left(P 2^{*}\right) \underset{(x, u) \in \mathbb{F}^{*}}{\operatorname{Minimize}} \max _{1 \leq i \leq r} \int_{a}^{b}\left\{f_{i}(x(t), u(t), t)+\left[x(t)^{T} E_{i}(t) x(t)\right]^{1 / 2}+\left[u(t)^{T} F_{i}(t) u(t)\right]^{1 / 2}\right\} d t ;$

$$
\underset{(x, u) \in \mathbb{F}^{*}}{\operatorname{Minimize}} \int_{a}^{b}\left\{f_{1}(x(t), u(t), t)+\left[x(t)^{T} E_{1}(t) x(t)\right]^{1 / 2}+\left[u(t)^{T} F_{1}(t) u(t)\right]^{1 / 2}\right\} d t,
$$

where $\mathbb{F}^{*}=\left\{(x, u) \in C^{n}[a, b] \times P W S^{m}[a, b]:(62)-(64)\right.$ hold $\}$ is the feasible set of $\left(\mathrm{P}^{*}\right)$.

To see more explicitly the modifications resulting from specializing the parametric optimality conditions of Section 3, we next combine, alter, and restate Theorems 3.1 and 3.2 for $\left(\mathrm{P}^{*}\right)$. We assume that the constraints of $\left(\mathrm{P}^{*}\right)$ satisfy Slater's constraint qualification (see Theorem 2.1).

TheOREM 7.1. A feasible solution $\left(x^{*}, u^{*}\right)$ of $\left(P^{*}\right)$ is optimal if and only if there exist $\lambda^{*} \in \Lambda, \mu^{*} \in \mathbb{R}_{+}, v^{*} \in P W S^{n}[a, b], w^{*} \in P W S_{+}^{s}[a, b]$, $\theta^{* i}, \xi^{* i} \in P W S^{n}[a, b], \pi^{* i}, \rho^{* i} \in P W S^{m}[a, b], i \in \underline{r}, \sigma^{* j} \in P W S^{n}[a, b]$, $\omega^{* j} \in P W S^{m}[a, b], j \in \underline{s}$, such that the following relations hold for all $t \in[a, b]:$

$$
\begin{aligned}
& \sum_{i=1}^{r} \lambda_{i}^{*}\left\{\nabla_{1} f_{i}\left(x^{*}, u^{*}, t\right)+E_{i}(t) \theta^{* i}(t)-\mu^{*}\left[\nabla_{1} g_{i}\left(x^{*}, u^{*}, t\right)-G_{i}(t) \xi^{* i}(t)\right]\right\} \\
& +A(t)^{T} v^{*}(t)+\sum_{j=1}^{s} w_{j}^{*}(t)\left[\nabla_{1} h_{j}\left(x^{*}, u^{*}, t\right)+R_{j}(t) \sigma^{* j}(t)\right]+D v^{*}(t)=0
\end{aligned}
$$




$$
\begin{aligned}
& \sum_{i=1}^{r} \lambda_{i}^{*}\left\{\nabla_{2} f_{i}\left(x^{*}, u^{*}, t\right)+F_{i}(t) \pi^{* i}(t)-\mu^{*}\left[\nabla_{2} g_{i}\left(x^{*}, u^{*}, t\right)-H_{i}(t) \rho^{* i}(t)\right]\right\} \\
& +B(t)^{T} v^{*}(t)+\sum_{j=1}^{s} w_{j}^{*}(t)\left[\nabla_{2} h_{j}\left(x^{*}, u^{*}, t\right)+S_{j}(t) \omega^{* j}(t)\right]=0 \\
& \sum_{j=1}^{s} w_{j}^{*}(t)\left\{h_{j}\left(x^{*}, u^{*}, t\right)+\left[x^{* T} R_{j}(t) x^{*}\right]^{1 / 2}+\left[u^{* T} S_{j}(t) u^{*}\right]^{1 / 2}\right\}=0 \\
& \int_{a}^{b} \sum_{i=1}^{r} \lambda_{i}^{*}\left\{f_{i}\left(x^{*}, u^{*}, t\right)+\left[x^{* T} E_{i}(t) x^{*}\right]^{1 / 2}+\left[u^{* T} F_{i}(t) u^{*}\right]^{1 / 2}\right. \\
& \left.-\mu^{*}\left\{g_{i}\left(x^{*}, u^{*}, t\right)-\left[x^{* T} G_{i}(t) x^{*}\right]^{1 / 2}-\left[u^{* T} H_{i}(t) u^{*}\right]^{1 / 2}\right\}\right\} d t=0, \\
& \theta^{* i}(t)^{T} E_{i}(t) \theta^{* i}(t) \leq 1, \quad \pi^{* i}(t)^{T} F_{i}(t) \pi^{* i}(t) \leq 1, \quad \xi^{* i}(t)^{T} G_{i}(t) \xi^{* i}(t) \leq 1, \\
& \rho^{* i}(t)^{T} H_{i}(t) \rho^{* i}(t) \leq 1, \quad i \in \underline{r} \text {, } \\
& \sigma^{* j}(t)^{T} R_{j}(t) \sigma^{* j}(t) \leq 1, \omega^{* j}(t)^{T} S_{j}(t) \omega^{* j}(t) \leq 1, j \in \underline{s} \\
& \theta^{* i}(t)^{T} E_{i}(t) x^{*}=\left[x^{* T}(t) E_{i} x^{*}\right]^{1 / 2}, \pi^{* i}(t)^{T} F_{i}(t) u^{*}=\left[u^{* T} F_{i}(t) u^{*}\right]^{1 / 2}, \\
& \xi^{* i}(t)^{T} G_{i}(t) x^{*}=\left[x^{* T} G_{i}(t) x^{*}\right]^{1 / 2}, \quad \rho^{* i}(t)^{T} H_{i}(t) u^{*}=\left[u^{* T} H_{i}(t) u^{*}\right]^{1 / 2}, \quad i \in \underline{r}, \\
& \sigma^{* j}(t)^{T} R_{j}(t) x^{*}=\left[x^{* T} R_{j}(t) x^{*}\right]^{1 / 2}, \omega^{* j}(t)^{T} S_{j}(t) u^{*}=\left[u^{* T} S_{j}(t) u^{*}\right]^{1 / 2}, \quad j \in \underline{s} .
\end{aligned}
$$

In a similar manner, one can readily specialize and restate Theorems

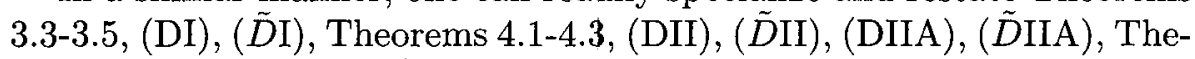

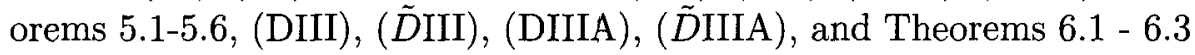
for $\left(\mathrm{P}^{*}\right),\left(\mathrm{P}^{*} 1\right),\left(\mathrm{P}^{*} 2\right)$, and $\left(\mathrm{P}^{*} 3\right)$.

Mathematical programming problems containing square roots of quadratic forms have been the subject of numerous investigations. These problems have arisen in stochastic programming, multifacility location problems, and portfolio selection problems, among others. Many optimality and duality results for several classes of these problems have appeared in the related literature. A fairly long list of references pertaining to various aspects of these problems is given in [26] (see also [4, $6,27,28]$ ). 


\section{References}

[1] D. J. Ashton, and D. R. Atkins, Multicriteria programming for financial planning, J. Oper. Res. Soc. Japan 30 (1979), 259-270.

[2] I. Barrodale, Best rational approximation and strict quasiconvexity, SIAM J. Numer. Anal. 10 (1973), 8-12.

[3] I. Barrodale, M. J. D. Powell and F. D. K. Roberts, The differential correction algorithm for rational $\ell_{\infty}$-approximation, SIAM J. Numer. Anal. 9 (1972), 493504.

[4] S. Chandra, B. D. Craven and I. Husain, A class of nondifferentiable control problems, J. Optim. Theory Appl. 56 (1985), 227-243.

[5] _ Continuous programming containing arbitrary norms, J. Aust. Math. Soc. 39 (1985), 28-38.

[6] _ A class of nondifferentiable continuous programming problems, J. Math. Anal. Appl. 107 (1985), 122-131.

[7] A. Charnes and W. W. Cooper, Goal programming and multiobjective optimization, part 1, European J. Oper. Res. 1 (1977), 39-54.

[8] B. D. Craven, (1988) Fractional Programming, Heldermann Verlag, Berlin, 1988.

[9] V. F. Demyanov and V. N. Malozemov, Introduction to Minimax, Wiley, New York, 1974.

[10] W. Dinkelbach, On nonlinear fractional programming, Management Sci. 13 (1967), 492-498.

[11] I. Ekeland and R. Temam, Convex Analysis and Variational Problems, NorthHolland, Amsterdam, 1976.

[12] R. A. Horn and C. R. Johnson, Matrix Analysis, Cambridge University Press, New York, 1985.

[13] J. S. H. Kornbluth, A survey of goal programming, Omega 1 (1973), 193-205.

[14] W. Krabs, Dualität bei diskreter rationaler Approximation, Internat. Ser. Numer. Math. 7 (1967), 33-41.

[15] J. V. Outrata and J. Jarušek, Duality in mathematical programming and optimal control, Kybernetika 20 (1984), 1-119.

[16] R. T. Rockafellar, Conjugate functions in optimal control and the calculus of variations, J. Math. Anal. Appl. 32 (1970), 174-222.

[17] _ Duality in optimal control, Lecture Notes in Mathematics, Vol. 680, Springer-Verlag, Berlin, 1978, pp. 219-257.

[18] I. M. Stancu-Minasian, Fractional Programming : Theory, Methods, and Applications, Kluwer Academic Publishers, Boston, 1977.

[19] M. B. Subrahmanyam, On applications of control theory to integral inequalities, J. Math. Anal. Appl. 77 (1980), 47-59.

[20] - Optimal Control with a Worst-Case Performance Criterion and Applications, Lecture Notes in Control and Inform. Sci. 145, Springer-Verlag, Berlin, 1990.

[21] _ Synthesis of finite-interval $H_{\infty}$ controllers by state-space methods, J. Guidance Control Dynamics 13 (1990), 624-627.

[22] _ Optimal disturbance rejection and performance robustness in linear systems, J. Math. Anal. Appl. 164 (1992), 130-150.

[23] J. Von Neumann, A model of general economic equilibrium, Rev. Econom. Stud. $13(1945), 1-9$. 
[24] J. Werner, Duality in generalized fractional programming, Internat. Ser. Numer. Math. 84 (1988), 341-351.

[25] G. J. Zalmai, A transposition theorem with applications to optimal control problems, Optimization 29 (1989), 265-279.

[26] Optimality conditions and duality models for a class of nonsmooth constrained fractional variational problems, Optimization 30 (1994), 15-51.

[27] _ _ Proper efficiency conditions and duality models for a class of constrained multiobjective fractional optimal control problems containing arbitrary norms, J. Optim. Theory Appl. 90 (1996), 435-456.

[28] __ Optimality conditions and duality models for a class of nonsmooth constrained fractional optimal control problems, J. Math. Anal. Appl. 210 (1997), 114-149.

[29] __ Saddle-point-type optimality conditions and Lagrangian-type duality for a class of constrained generalized fractional optimal control problems, Optimization 44 (1998), 351-372.

[30] __ Optimality principles and duality models for a class of continuous-time generalized fractional programming problems with operator constraints, J. Stat. Manag. Syst. 1 (1998), 61-100.

Department of Mathematics and Computer Science

Northern Michigan University

Marquette, Michigan 49855, USA

E-mail: gzalmai@nmu.edu 